\title{
Steady-state and equilibrium vortex configurations, transitions, and evolution in a mesoscopic superconducting cylinder
}

\author{
Sangbum Kim, ${ }^{1}$ Chia-Ren $\mathrm{Hu}^{2}$ and Malcolm J. Andrews ${ }^{1}$ \\ ${ }^{1}$ Department of Mechanical Engineering, Texas A\&M University, College Station, Texas 77843, USA \\ ${ }^{2}$ Center for Theoretical Physics, Department of Physics, Texas A\&M University, College Station, Texas 77843, USA
}

(Received 15 January 2003; revised manuscript received 18 November 2003; published 29 March 2004)

A numerical scheme to study the mixed states in a mesoscopic type-II superconducting cylinder is described. Steady-state configurations and transient behavior of the magnetic vortices for various values of the applied magnetic field $H$ are presented. Transitions between different multivortex states as $H$ is changed is demonstrated by contour plots and jumps in the $B$ vs $H$ plot. We evolve a uniformly superconducting initial state using the simplest set of relaxation equations. The results reveal that the system passes through nearly metastable intermediate configurations while seeking the final minimum-energy steady state consistent with the square symmetry of the sample. An efficient scheme to determine the equilibrium vortex configuration in a mesoscopic system at any given applied field, not limited to the symmetry of the system, is devised and demonstrated.

DOI: 10.1103/PhysRevB.69.094521

PACS number(s): 74.78.Na, 74.25.Qt, 74.25.Op

\section{INTRODUCTION}

The electromagnetic characteristics of type-II superconductors have been of great interest, particularly since the discovery of high-temperature superconductors. Furthermore, a recent surge in interest about nanotechnology has drawn the attention of researchers from various disciplines to a detailed understanding of the characteristics of mesoscopic superconducting samples. Several phenomenological theories have been developed during decades of superconductor research, with one popular choice being the GinzburgLandau theory. ${ }^{1,2}$ Its time-dependent extension is known as the time-dependent Ginzburg-Landau (TDGL) theory. ${ }^{3}$

In the Ginzburg-Landau theory, the electromagnetic state of a superconductor can be determined by solving a system of partial differential equations. Abrikosov discovered that if the $\kappa$ parameter, now known as the Ginzburg-Landau parameter, is larger than $1 / \sqrt{2}$, then when a bulk superconductor is placed in a sufficiently large magnetic field, the magnetic field penetrates the superconductor in the form of singly quantized vortices. Around each vortex flows a supercurrent, ${ }^{4}$ confining a single quantum of magnetic flux within it. Superconductors with this property are known as type-II.

Here we present the result of a numerical study about the magnetization process inside a superconducting square cylinder, with submicron lateral dimension in an external magnetic field. We have restricted the work to a square cross section of a linear size equal to 4.65 times $\lambda$ (the magnetic penetration depth). Taking $\lambda$ at $500 \AA$, then the crosssectional area is $0.054 \mu \mathrm{m}^{2}$.

Previous works on the magnetization of a mesoscopic superconductor without pinning centers have been reported by Peeters $e t a^{5-8}$ and others, ${ }^{9,10}$ who presented extensive calculations on the superconducting state in mesoscopic, type-I, superconducting thin films. In most cases they found transitions between giant vortex states of different circulation quantum numbers $L$, with some multivortex states occasion- ally appearing as thermodynamically stable states, but mostly as metastable states. These predictions appear to have already received some level of experimental confirmation, although some discrepancies still exist. ${ }^{11}$ (Reference 10 mainly compared the energy of a "3-2" vortex-antivortex molecule state with that of a single off-centered vortex state at $L=1$, as both evolve to the equilibrium state of a single vortex at the center.) Misko et al. ${ }^{12}$ studied both type-I and type-II mesoscopic triangular cylinders, and have shown that a vortex-antivortex molecule appears only if the sample is type-I. They considered only one field value at which $L=2$ is favored, and did not consider vortex configurational transitions as the field changes. ${ }^{13}$

Our aim is to simulate how vortices enter and settle in stable arrangements when a mesoscopic type-II superconductor of a given symmetry is first cooled below the critical temperature, and then an external magnetic field is applied. This is often termed zero-field cooling (ZFC). We find that only vortex numbers and configurations consistent with the sample symmetry can appear in this case. It is known that global minimum-energy vortex configurations exist with reduced symmetry, with corresponding final equilibrium states at general values of the applied field. To find these equilibrium states we developed an efficient numerical scheme.

Our approach is to solve a set of simplified (and discretized) TDGL equations, in which the coupling to the electric field is neglected, and the superconducting order parameter and the magnetic field are assumed to relax with the same time scale. These assumptions are not physical, but are acceptable here, since we are only interested in obtaining the final steady-state vortex configurations, and the symmetryrelated qualitative behavior of the transient configurations and their evolution. For a more physical set of TDGL equations see the work of Tinkham. ${ }^{3}$ For an example of the numerical solution of such a set of TDGL equations, see Ref. 14. Our numerical method may be understood to be a relaxation procedure with a pseudotime. ${ }^{15,16}$ Since the equations we have solved do not contain any thermal fluctuation terms, and the sample we considered has a perfect square symmetry, 
we find that when starting with the Meissner state and no field penetration, the final steady-state vortex configurations we obtain all have perfect square symmetry, with vortex numbers also limited to only multiples of 4 (the symmetry number). These configurations would correspond to physical situations under zero-field cooling, if the physical sample has perfect symmetry, and the temperature is sufficiently low, so that thermal fluctuations are not able to overcome any energy barrier for vortex entry, expulsion, or rearrangement. If the sample surface has slight imperfection, or if the temperature is not sufficiently low, then these configurations are, in most cases, not in equilibrium at the given magnetic-field strength. Even the vortex number may not be correct; however, if we insert terms to simulate thermal fluctuation into the equations, as in the method of simulated annealing, ${ }^{17,18}$ then the simulation computer program will take a much longer time to run, and may become impractical even with a supercomputer. We have devised an efficient scheme to find the equilibrium vortex configurations: We solve the same set of relaxation equations without any thermal fluctuation terms, but instead of starting the solution with the Meissner state as the initial state, we devise artificial initial states with a given number of vortices in random positions. We present analytic expressions for such initial states in terms of a widely known approximate expression for a singly quantized vortex in cylindrical coordinates. Then, for vortex numbers not too different from the equilibrium number, the final steady states obtained by solving our relaxation equations will, in most cases, have the number of vortices close to those of the initial states. By comparing the total Gibbs energies of these steady states with different vortex numbers we can find the state with the lowest total Gibbs energy, which we identify as the equilibrium state with the equilibrium vortex number. Sometimes we obtain more than one configuration for the same vortex number (when the vortex number exceeds four), then their Gibbs energies are also compared. We give an explicit demonstration of this scheme, ${ }^{19}$ which might be very useful in view of the recent interest in nanoscience and nanotechnology. ${ }^{20}$ We note that in a bulk sample vortices like to form a triangular lattice. Thus, when the sample does not conform with this symmetry, and if the sample is sufficiently small so that the boundary effect on the equilibrium vortex configurations is important, then the system is frustrated, and the equilibrium vortex configurations can be quite intriguing and difficult to foresee.

\section{THE SIMPLIFIED TIME-DEPENDENT GINZBURG-LANDAU MODEL}

In an external magnetic field $\mathbf{H}$, the Gibbs free-energy density $g$ of a superconducting state is given by: ${ }^{3}$

$$
\begin{aligned}
g= & f_{n}+\alpha|\Psi|^{2}+\frac{\beta}{2}|\Psi|^{4}+\frac{1}{2 m_{s}}\left|\left(-\imath \hbar \nabla-\frac{e_{s}}{c} \mathbf{A}\right) \Psi\right|^{2} \\
& +\frac{|\mathbf{h}|^{2}}{8 \pi}-\frac{\mathbf{h} \cdot \mathbf{H}}{4 \pi} .
\end{aligned}
$$

Here, $f_{n}$ is the free-energy density in the normal state in the absence of the magnetic field; $\Psi$ is the complex-valued order parameter, with the superscript $*$ denoting complex conjugation; $\mathbf{A}$ is the magnetic vector potential, $\mathbf{h}=\boldsymbol{\nabla} \times \mathbf{A}$ denotes the induced magnetic field, and $\mathbf{H}$ represents the applied magnetic field. The supercurrent density is expressed as $\quad \mathbf{J}_{s}=\boldsymbol{\nabla} \times \boldsymbol{\nabla} \times \mathbf{A}=\left(e_{s} \hbar / 2 \imath m_{s}\right)\left(\Psi^{*} \boldsymbol{\nabla} \Psi-\Psi \boldsymbol{\nabla} \Psi^{*}\right)-\left(e_{s}^{2} /\right.$ $\left.m_{s} c\right)|\Psi|^{2} \mathbf{A}$. In the above, $e_{s}$ is the "effective charge" of a Cooper pair which is twice the charge of an electron, and $m_{s}$ its "effective mass" which can be selected arbitrarily, but the conventional choice is twice the mass of an electron. Also, $c$ is the speed of light, and $\hbar=h / 2 \pi$ where $h$ is Planck's constant.

Ginzburg-Landau theory postulates that the Gibbs free energy $G$ of a superconducting sample $\Omega$ is at a minimum in the superconducting state. The celebrated Ginzburg-Landau equations are obtained by minimizing this functional with respect to $\Psi$ and $\mathbf{A}$ using the variational principle.

Since a constant term does not change the end result of the variational technique, an algebraic manipulation is made to subtract $f_{n}$ and add $\mathbf{H} \cdot \mathbf{H} / 8 \pi$ to the $g$ above, giving: ${ }^{21}$

$$
\begin{aligned}
G(\Psi, \mathbf{A})= & \int_{\Omega}\left(\alpha|\Psi|^{2}+\frac{\beta}{2}|\Psi|^{4}+\frac{|\mathbf{h}-\mathbf{H}|^{2}}{8 \pi}\right. \\
& \left.+\frac{1}{2 m_{s}}\left|\left(-\imath \hbar \nabla-\frac{e_{s}}{c} \mathbf{A}\right) \Psi\right|^{2}\right) d \Omega .
\end{aligned}
$$

We introduce dimensionless variables as follows:

$$
\begin{gathered}
x^{\prime}=\frac{x}{\lambda}, \mathbf{H}^{\prime}=\frac{\mathbf{H}}{\sqrt{2} H_{c}}, \quad \mathbf{h}^{\prime}=\frac{\mathbf{h}}{\sqrt{2} H_{c}}, \\
\mathbf{j}^{\prime}=\frac{2 \sqrt{2} \pi \lambda}{c H_{c}} \mathbf{j}, \quad \mathbf{A}^{\prime}=\frac{\mathbf{A}}{\sqrt{2} H_{c} \lambda}, \quad \Psi^{\prime}=\frac{\Psi}{\Psi_{0}} .
\end{gathered}
$$

The characteristic scales are: $\left|\Psi_{0}\right|=\sqrt{-\alpha / \beta}$, which is the magnitude of $\Psi$ that minimizes the free energy in the absence of a field; the thermodynamic critical field strength $H_{c}=\left(4 \pi|\alpha|\left|\Psi_{0}\right|^{2}\right)^{1 / 2}$, which divides the normal state and superconducting state regions in type-I superconductor phase diagram; the London penetration depth $\lambda=\left(m_{s} c^{2} / 4 \pi\left|\Psi_{0}\right|^{2} e_{s}^{2}\right)^{1 / 2} ; \quad$ the coherence length $\xi$ $=\left(\hbar^{2} / 2 m_{s}|\alpha|\right)^{1 / 2} ;$ and, the Ginzburg-Landau parameter $\kappa$ $=\lambda / \xi$.

We obtain the dimensionless gauge-invariant free-energy functional, omitting primes for convenience.

$$
\begin{aligned}
G(\Psi, \mathbf{A})= & \int_{\Omega}\left(-|\Psi|^{2}+\frac{1}{2}|\Psi|^{4}+|\boldsymbol{\nabla} \times \mathbf{A}-\mathbf{H}|^{2}\right. \\
& \left.+\left|\left(\frac{\boldsymbol{\nabla}}{\kappa}-\imath \mathbf{A}\right) \Psi\right|^{2}\right) d \Omega .
\end{aligned}
$$

The simplified TDGL model we employ to find solutions of the static GL equations may be viewed as a gradient flow of the energy functional. That is, the variation of $(\Psi, \mathbf{A})$ with respect to time should be in the opposite direction of the gradient of the energy functional, $(\partial \Psi / \partial t)=-\left(\partial G / \partial \Psi^{*}\right)$, $(\partial \mathbf{A} / \partial t)=-\frac{1}{2}(\partial G / \partial \mathbf{A})$ with time $t$ in units of the only 


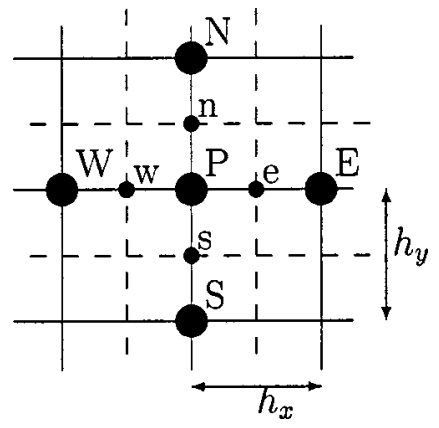

FIG. 1. The staggered grid arrangement for cell nodes $P, E, W, N, S$ and faces $e, w, n, s$.

relaxation time of the equations. The natural boundary conditions are: (1) the continuity of the parallel component of the magnetic field across the boundary surface: $(\boldsymbol{\nabla} \times \mathbf{A}) \times \mathbf{n}$ $=\mathbf{H} \times \mathbf{n}$, (for two-dimensional problems only, see below), and (2), the vanishing gauge-invariant normal derivative of $\Psi:(\boldsymbol{\nabla} / \kappa-\imath \mathbf{A}) \Psi \cdot \mathbf{n}=0,{ }^{22}$ with $\mathbf{n}$ denoting the outward surface normal.

\section{DISCRETIZATION AND CALCULATION PROCEDURE}

For long square cylindrical samples, we need only to solve a two-dimensional (2D) problem. We take $\mathbf{A}$ $=[A(x, y), B(x, y), 0]$ and $\mathbf{H}=(0,0, H)$, where $H=(\boldsymbol{\nabla} \times \mathbf{A})_{z}$ $=(\partial B / \partial x)-(\partial A / \partial y)$.

Defining the link variables as below, the gauge invariance is preserved in discretizing the Gibbs free energy and the consequent simplified-TDGL equations. Defining $W(x, y)$ $\left.=\exp \left[\imath \kappa \int^{x} A(s, y) d s\right] ; \quad V(x, y)=\exp \left[\imath \kappa \int^{y} B(x, \eta) d \eta\right]\right)$ and noting that $\left|\partial_{x}\left(W^{*} \Psi\right)\right|=\left|\left(\partial_{x}-\imath \kappa A\right) \Psi\right|$, and $\left|\partial_{y}\left(V^{*} \Psi\right)\right|=\mid\left(\partial_{y}\right.$ $-\imath \kappa B) \Psi \mid$, we have:

$$
\begin{aligned}
G(\Psi, \mathbf{A})= & \int_{\Omega}\left(-|\Psi|^{2}+\frac{1}{2}|\Psi|^{4}+|\boldsymbol{\nabla} \times \mathbf{A}-\mathbf{H}|^{2}\right. \\
& \left.+\left|\frac{1}{\kappa} \partial_{x}\left(W^{*} \Psi\right)\right|^{2}+\left|\frac{1}{\kappa} \partial_{y}\left(V^{*} \Psi\right)\right|^{2}\right) d \Omega .
\end{aligned}
$$

We discretize the free-energy functional on a staggered grid over $\Omega$ shown in Fig. 1. ${ }^{23,16}$ This gives us a secondorder approximation in $h_{x}$ and $h_{y}$ to the continuous energy functional, where the $h_{x}$ and $h_{y}$ are the spatial increments in the $x$ direction and $y$ direction. The staggered grid also leads to a satisfactory way of discretizing the natural boundary conditions. ${ }^{24}$ For a rectangular grid, the first component of the vector potential is constant in time on one pair of the edges of the boundary, and the second component is constant in time on the other pair. ${ }^{22}$

In this paper, we assume that the cylindrical superconductor has a square cross section and is subject to an applied field along the central axis. The applied field is assumed to be constant in time. We further assume the order parameter $\Psi$ varies in the cross-sectional plane of the square cylindrical sample, and the vector potential A has only two nonzero components $(A, B)$, which also lie in this plane. We also assume that the superconductor has no pinning sites. Then at steady-state conditions, the vortices settle at maximal dis- tances due to mutual repulsion. This requirement leads to a triangular lattice of vortices in an infinitely large domain. ${ }^{3}$

In the staggered grid the lattice evaluation points for $\Psi$, $A$, and $B$ are all different, with $\Psi$ evaluated at the node center $(i, j), A$ evaluated at the east cell face $(i+1 / 2, j)$, and $B$ evaluated at the north cell face $(i, j+1 / 2)$. According to Refs. 23,16, this formulation keeps second-order accuracy in the derivative evaluations as they appear in each of the discretized equations.

The discrete equations are obtained by minimizing the discrete energy functional $G_{d}$ with respect to the variation in $\Psi$ and $\mathbf{A}$ as:

$$
\begin{aligned}
\frac{\partial \Psi_{P}}{\partial t}= & \frac{h_{x} h_{y}}{\kappa^{2}}\left(\frac{e^{l A_{w} \kappa h_{x}} \Psi_{W}-2 \Psi_{P}+e^{-\imath A_{e} \kappa h_{x}} \Psi_{E}}{h_{x}^{2}}\right. \\
& \left.+\frac{e^{\imath B_{s} \kappa h_{y}} \Psi_{S}-2 \Psi_{P}+e^{-\imath B_{n} \kappa h_{y}} \Psi_{N}}{h_{y}^{2}}\right)+h_{x} h_{y} N_{1}\left(\Psi_{P}\right) \\
\frac{\partial A_{e}}{\partial t}= & -h_{x}\left(\frac{B_{n E}-B_{n}+B_{s}-B_{s E}}{h_{x}}-\frac{A_{N e}-2 A_{e}+A_{S e}}{h_{y}}\right) \\
& +\frac{h_{y}}{\kappa} N_{2}\left(A_{e}, \Psi_{P}, \Psi_{E}\right), \\
\frac{\partial B_{n}}{\partial t}= & -h_{y}\left(\frac{A_{w}-A_{e}+A_{N e}-A_{N w}}{h_{y}}-\frac{B_{n E}-2 B_{n}+B_{n W}}{h_{x}}\right) \\
& +\frac{h_{x}}{\kappa} N_{3}\left(B_{n}, \Psi_{P}, \Psi_{N}\right),
\end{aligned}
$$

with

$$
\begin{aligned}
& N_{1}\left(\Psi_{P}\right)=\left(1-\left|\Psi_{P}\right|^{2}\right) \Psi_{P}, \\
& N_{2}\left(A_{e}, \Psi_{P}, \Psi_{E}\right)=\left(\Phi_{P} \Theta_{E}-\Theta_{P} \Phi_{E}\right) \cos \left(A_{e} \kappa h_{x}\right) \\
&-\left(\Phi_{P} \Phi_{E}+\Theta_{P} \Theta_{E}\right) \sin \left(A_{e} \kappa h_{x}\right), \\
& N_{3}\left(B_{n}, \Psi_{P}, \Psi_{N}\right)=\left(\Phi_{P} \Theta_{N}-\Theta_{P} \Phi_{N}\right) \cos \left(B_{n} \kappa h_{y}\right) \\
&-\left(\Phi_{P} \Phi_{N}+\Theta_{P} \Theta_{N}\right) \sin \left(B_{n} \kappa h_{y}\right),
\end{aligned}
$$

where $\Theta$ and $\Phi$ are the real and imaginary parts of $\Psi$, and boundary conditions of the computational domain $\Omega$ (with $T$, $B, L$, and $R$ denoting top, bottom, left, and right, respectively):

$$
\begin{gathered}
\Psi_{P}=\Psi_{S} e^{\imath \kappa h_{y} B_{s}} \quad \text { on } \Omega_{T}, \\
\Psi_{P}=\Psi_{N} e^{-\imath \kappa h_{y} B_{n}} \quad \text { on } \Omega_{B}, \\
\Psi_{P}=\Psi_{E} e^{-\imath \kappa h_{x} A_{e}} \quad \text { on } \Omega_{L}, \\
\Psi_{P}=\Psi_{W} e^{\imath \kappa h_{x} A_{w}} \text { on } \Omega_{R}, \\
A_{e}=A_{S e}-\left(H-\frac{B_{n E}-B_{n}}{h_{x}}\right) h_{y} \text { on } \Omega_{T},
\end{gathered}
$$




$$
\begin{aligned}
& A_{e}=A_{N e}+\left(H-\frac{B_{n E}-B_{n}}{h_{x}}\right) h_{y} \text { on } \Omega_{B}, \\
& B_{n}=B_{n E}-\left(H+\frac{A_{N e}-A_{e}}{h_{y}}\right) h_{x} \text { on } \Omega_{L}, \\
& B_{n}=B_{n W}+\left(H+\frac{A_{N e}-A_{e}}{h_{y}}\right) h_{x} \text { on } \Omega_{R} .
\end{aligned}
$$

The finite difference equations are solved by the Euler method with $h_{x}=h_{y}=0.15$ and $\Delta t=0.05$, and taking $\kappa=4$. In the numerical computations that follow, all details are kept the same except for the strength of the applied magnetic field and/or the initial conditions.

Extensive grid independence checks were performed during this work, and typical results are described in Sec. VI of this paper.

\section{STEADY STATES UNDER ZERO-FIELD COOLING IN A PERFECTLY SQUARE SAMPLE AT LOW TEMPERATURES}

We first solve the above set of equations assuming that the initial state is the perfect Meissner state with no field penetration. As explained in the introduction, this corresponds to applying a magnetic field after zero-field cooling. Figure 2 shows plots of $|\Psi|^{2}$ [the left figure in (a)-(k)] and $h=\nabla$ $\times \mathbf{A}$ [the right figure in $(\mathrm{a})-(\mathrm{k})]$ for the final steady states reached for a sequence of increasing $H$ values. In the left figures $|\Psi|^{2}$, which is interpreted physically as the density of Cooper pairs, runs from 0 to 1 , with level 1 corresponding to the full superconducting state. Each isolated group of contours is called a "vortex," representing the supercurrent $J$ circling around the vortex core, with $\Psi=0$ at the vortex core. In the 3D plots of Fig. 2(b), it is clear that the vortices reach close to $|\Psi|^{2}=0$ at the core.

We note that the number of vortices increases in multiples of 4 . This is a consequence of the fact that the vortices are symmetrically created at the midpoints of the sample edges. Perfect symmetry in the sample geometry dictates that each side creates an equal number of vortices. For sample sizes less than $\lambda$, it is to be expected that simultaneous penetration of four vortices is energetically unfavorable compared with a single vortex penetration. However, a single vortex entry is still prohibited if symmetry is strictly preserved. The magnetic field will simply penetrate the sample without entry of vortices in that case. When symmetry is not strictly preserved, and if the sample size is much smaller than $\lambda$, then no vortex will enter the sample since the magnetic field already penetrates the sample. If the sample size is of the order of $\lambda$, then one vortex will enter and move to its center. In these calculations, symmetry is closely preserved, and in this case, vortices enter in multiples of 4 for a square cross section. For a hexagonal cross section, it should be a multiple of 6. For a large circular cylinder, vortices of the usual kind (with line singularities) cannot enter. Rather, "cylindrical vortex sheets," with phase-winding quantum number $n$ about the axis, change from $n$ at radial coordinate $\rho-\epsilon$ to $n+1$ at $\rho+\epsilon$, and must be created at the boundary and then move radially inward. This is true only if symmetry is strictly preserved. However, this can only happen in an actual sample if the sample strictly obeys the symmetry, and the temperature is very close to absolute zero. Otherwise, vortices will enter one at a time via defect sites at the surface, or through thermal fluctuations to destroy the symmetry. Then it will not be a multiple of 4 for a square cross section, or a multiple of 6 for a hexagonal cross section, and the relationship between sample size and number of vortices becomes a less significant concern.

The symmetry here manifests itself in this geometrydominated problem, and vortices arrange themselves in the square-symmetric configurations. The resultant steady states are mostly not true equilibrium states, since the vortex arrangements do not reflect the intrinsic tendency of vortices to form a triangular lattice known to appear in bulk samples. The natural next step is to add a thermal fluctuation term to find the true equilibrium states which may or may not conform with this symmetry. Such an approach would then be like simulated annealing. ${ }^{17,19}$ We believe it would not be practical to perfect this approach since it will likely be difficult to determine the appropriate rate of cooling and the starting temperature. In addition the run time of the computer program might also be expected to be much longer than we have found here, so we have devised a different approach which we believe is much more efficient at finding the equilibrium states. This is given in a later section. We shall see that even the cases with a low number of vortices are not the true equilibrium. Also of interest is the fact that for various $H$, the vortex configuration requires much longer run times to get to a steady state. Geometry controls the settling time more than the energy in these cases.

Our results are summarized in Table I, which lists the range of $H$ for each resultant number of vortices, and the corresponding induced magnetic field $B=(1 /|\Omega|) \int_{\Omega} h d \Omega$. The Table shows that the final number of vortices does not change within various bands of the applied magnetic field $H$. The $H$ 's listed correspond to the threshold values (upper and lower limits) for each band. They were found on a trial-anderror basis, and can be refined to any desired accuracy. The Table and Fig. 2 show that, below $H=0.839$, there is no vortex. Between $H=0.84$ and 1.144 , the vortex number $n_{\infty}$ $=4$, and so on.

The $B$ vs $H$ plot shown in Fig. 3, reveals that $B$ is much lower than $H$ when the number of vortices is small, but as the vortices increase, the curve approaches the $B=H$ curve asymptotically. The figure also shows an abrupt increase in $B$ between the regions of different number of vortices. For example, when $H$ changes from 1.144 to $1.145, B$ changes from 0.9779 to 1.0694 . If the limit $\Delta H \rightarrow 0$ is taken, we expect a sudden configurational phase transition increasing the number of vortices, as is apparent in Figs. 2(c) and 2(d). Such mini-first-order transitions are known ${ }^{5-9}$ to occur in a mesoscopic superconductor as $H$ is changed, but the details are quite different, because different parameter $(\kappa)$ regimes and sample geometries (cylinder vs film) were studied.

Comparison of Fig. 2(g) with 2(h) reveals a phase transition is also evident for the $n_{\infty}=12$ case, where the vortex 

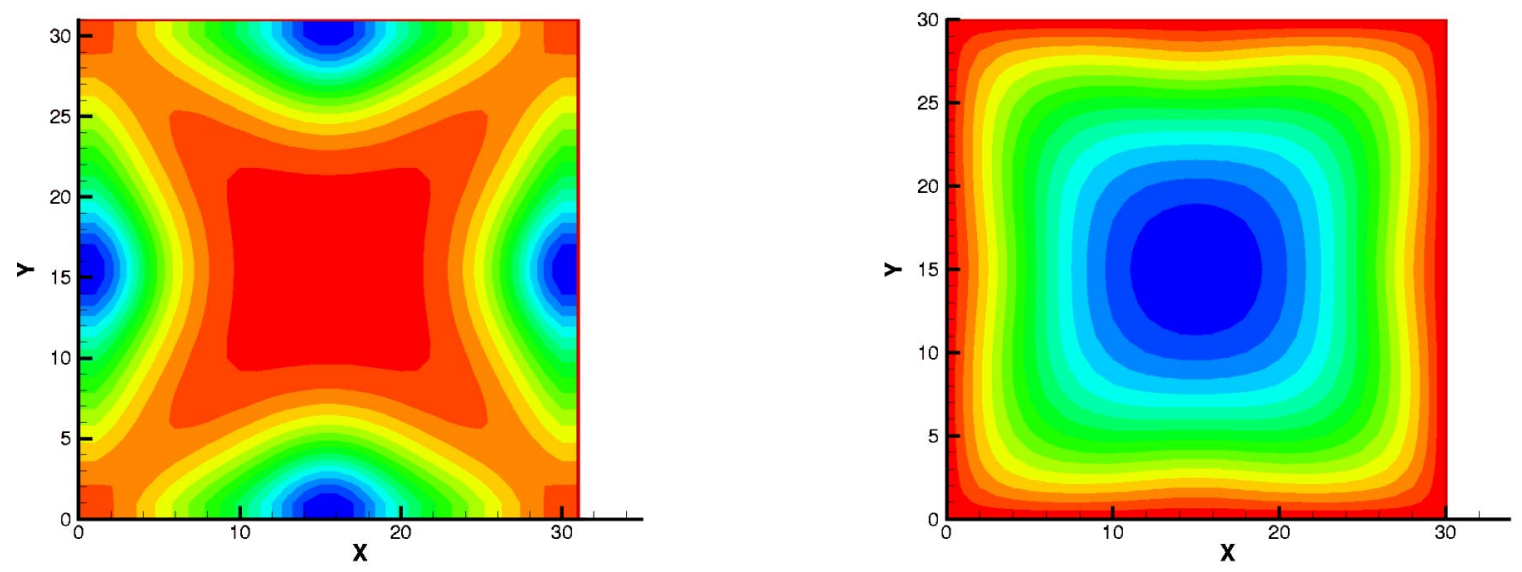

(a) $\mathrm{H}=\mathbf{0 . 8 3 9}$
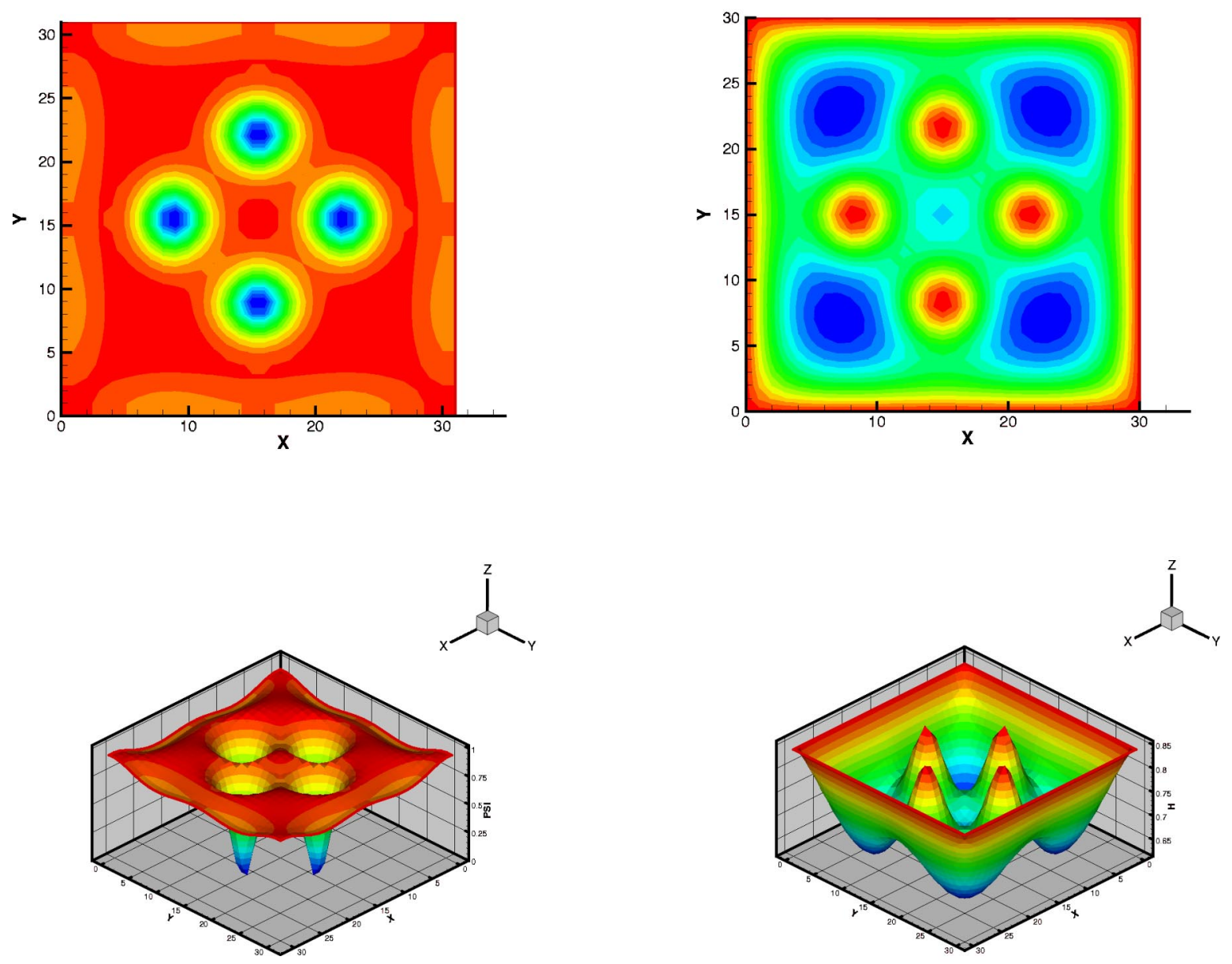

(b) $\mathrm{H}=\mathbf{0 . 8 4 0}$

FIG. 2. Plots of $|\Psi|^{2}$ and $h=\boldsymbol{\nabla} \times \mathbf{A}$ for various $H\left[|\Psi|^{2}\right.$ is shown on the left in (a)-(k), and $h$ on the right in (a)-(k)]. These final steady-state solutions are obtained from a uniform superconducting state. 

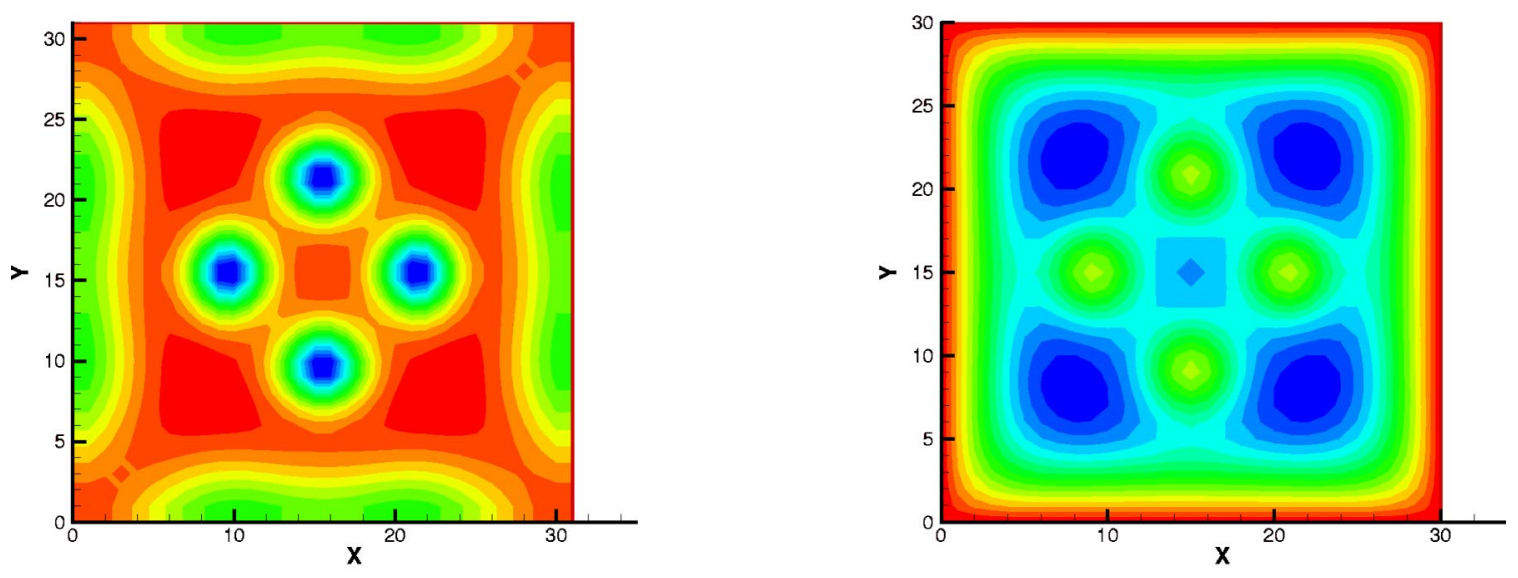

(c) $H=1.144$
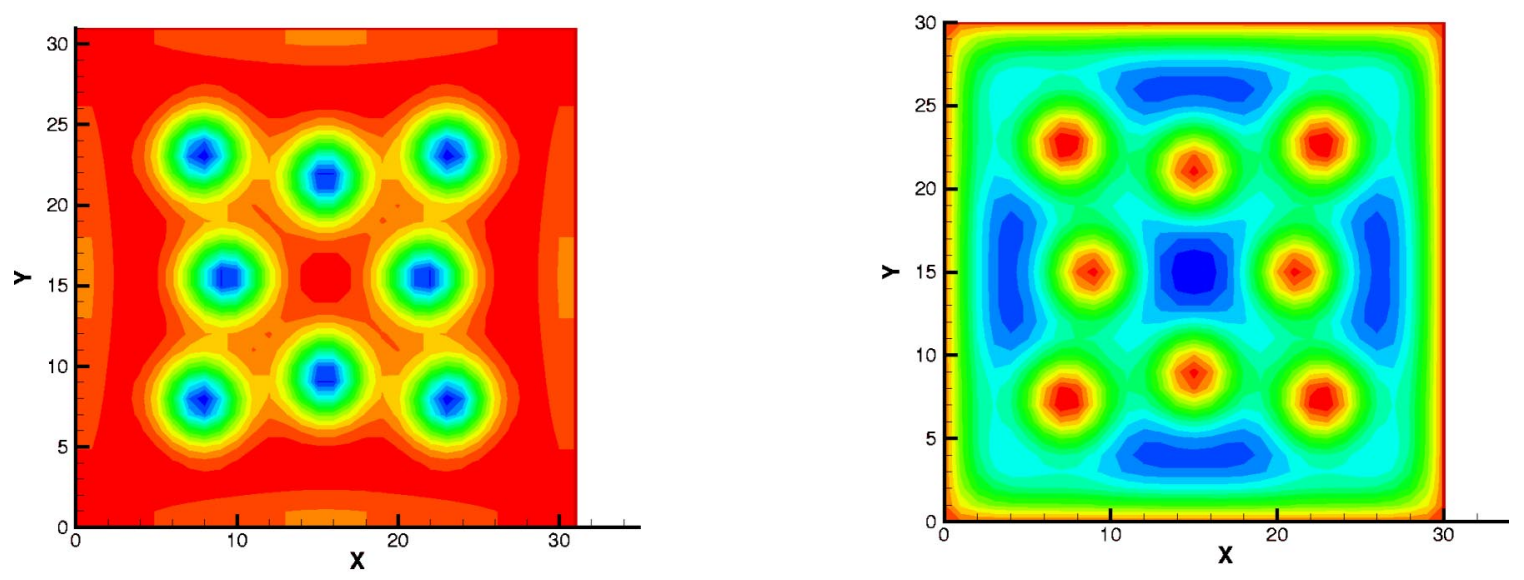

(d) $H=1.145$
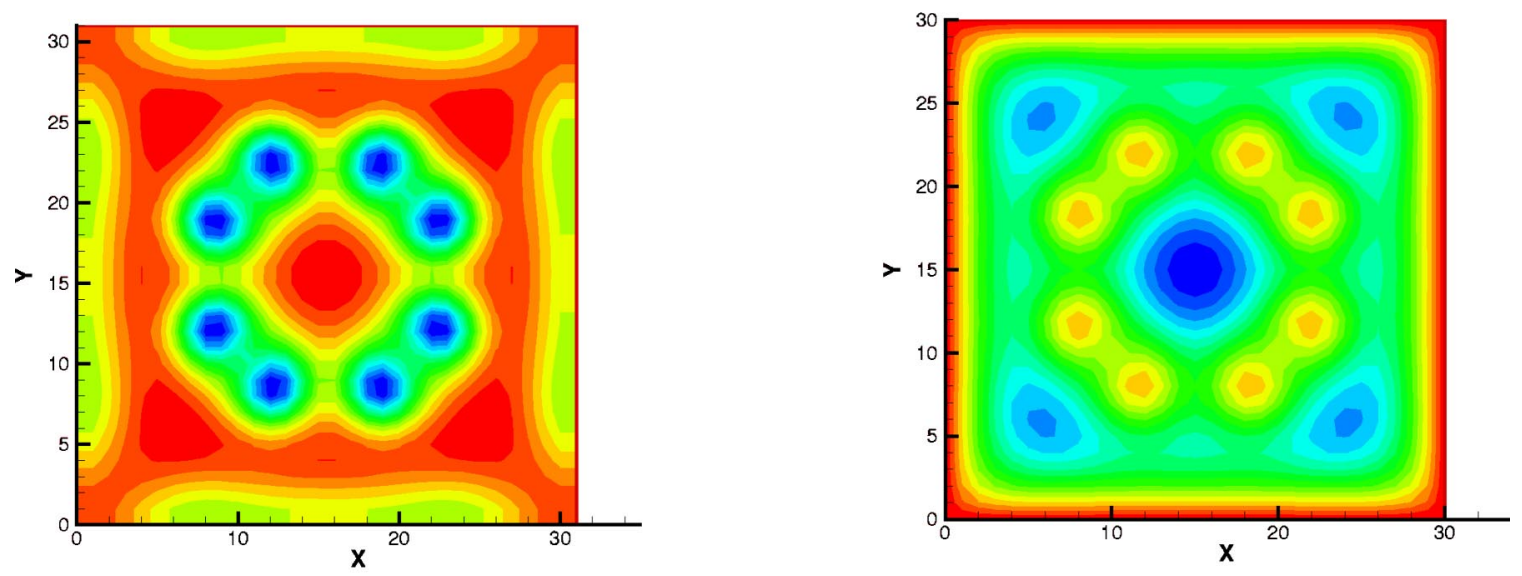

(e) $H=1.429$

FIG. 2. (Continued). 

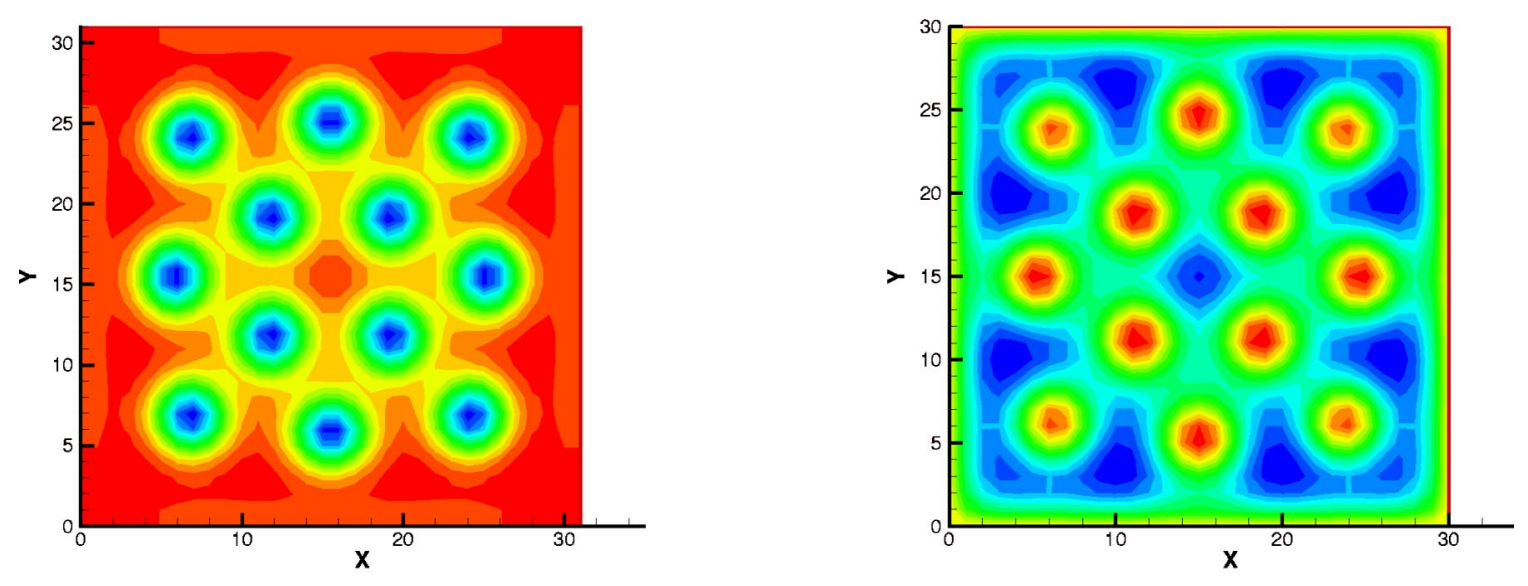

(f) $H=1.430$
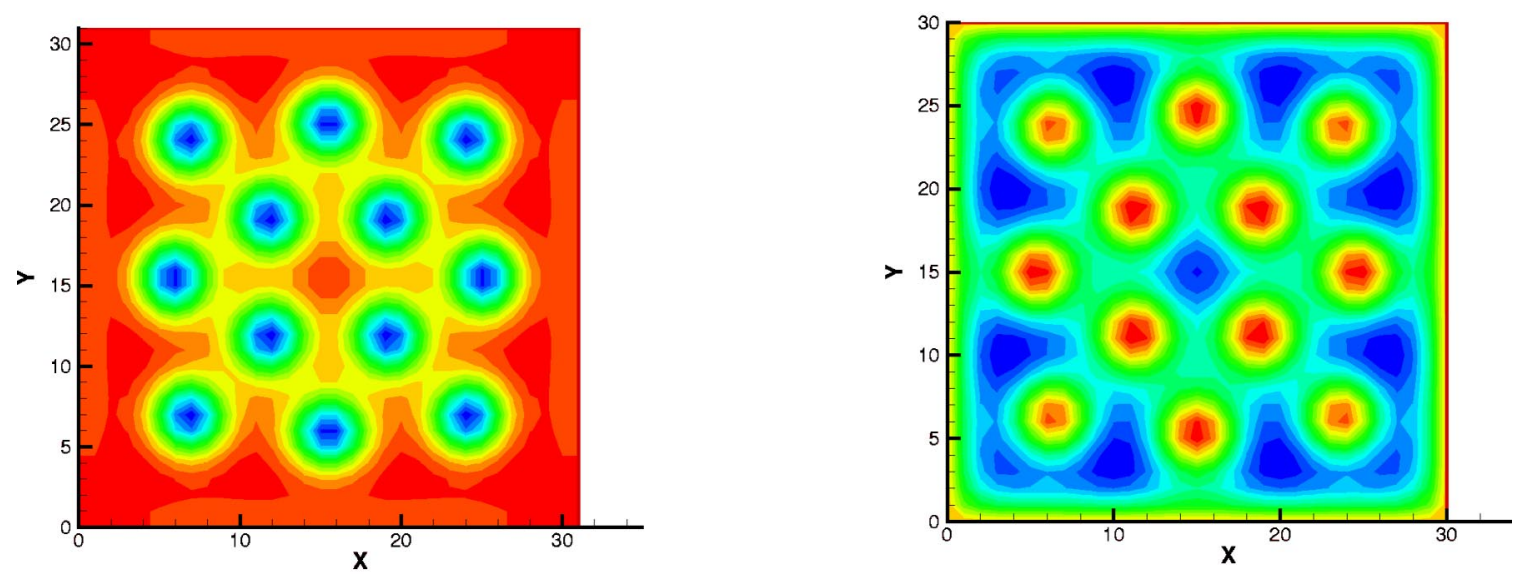

(g) $H=1.454$
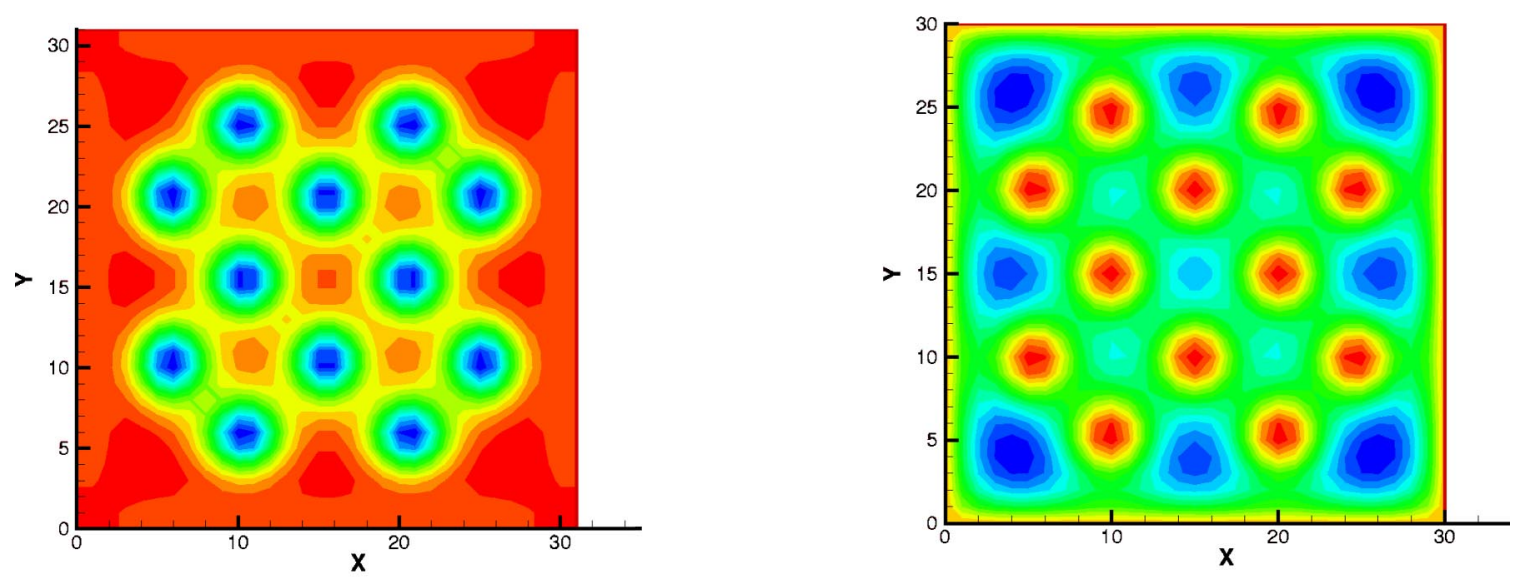

(h) $H=1.455$ 

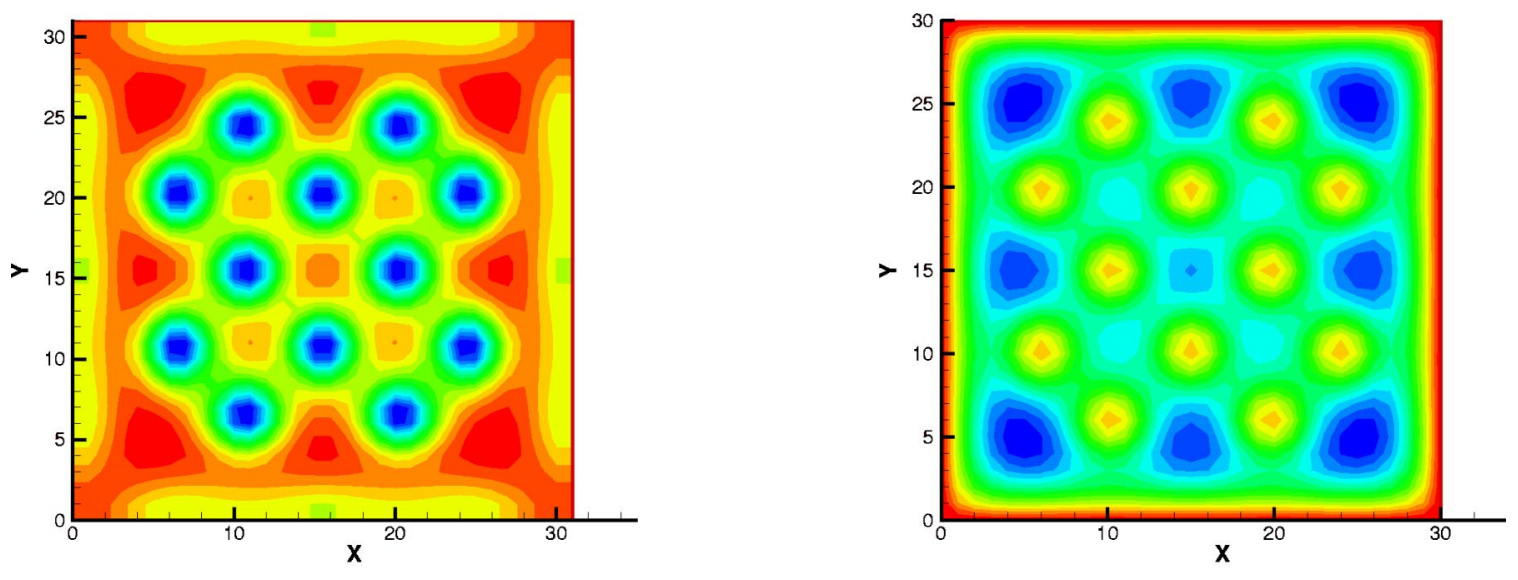

(i) $\mathrm{H}=1.732$
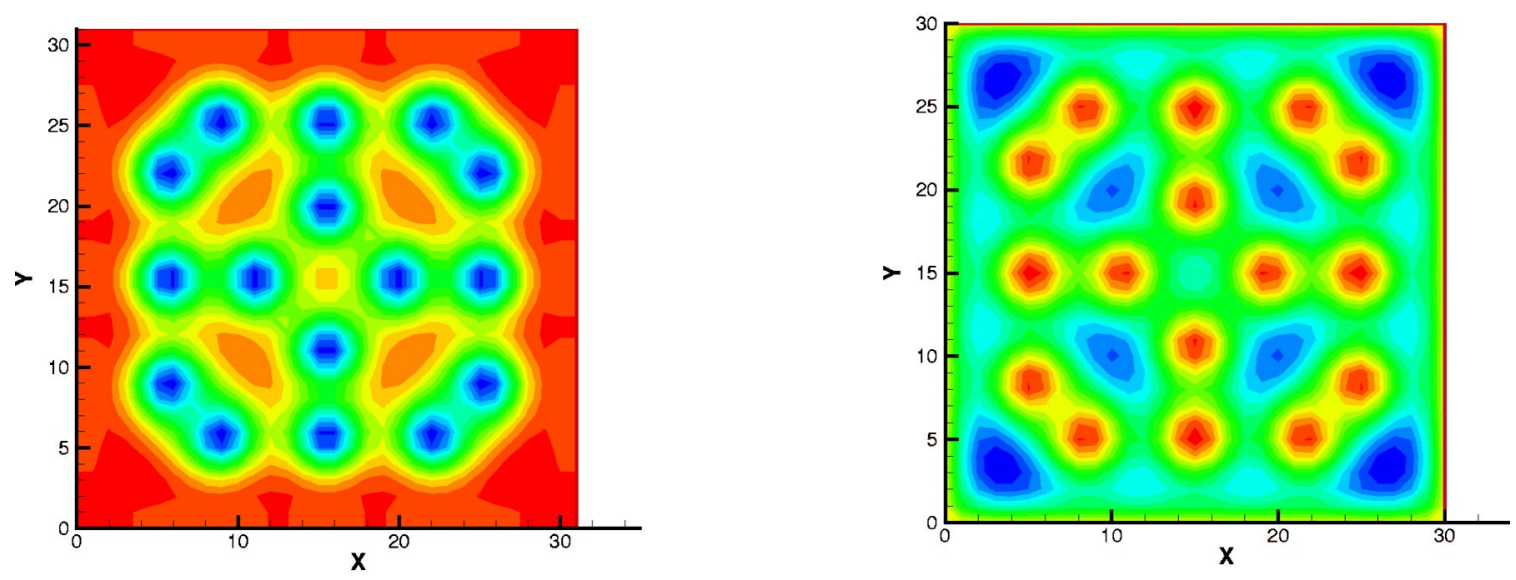

(j) $H=1.733$
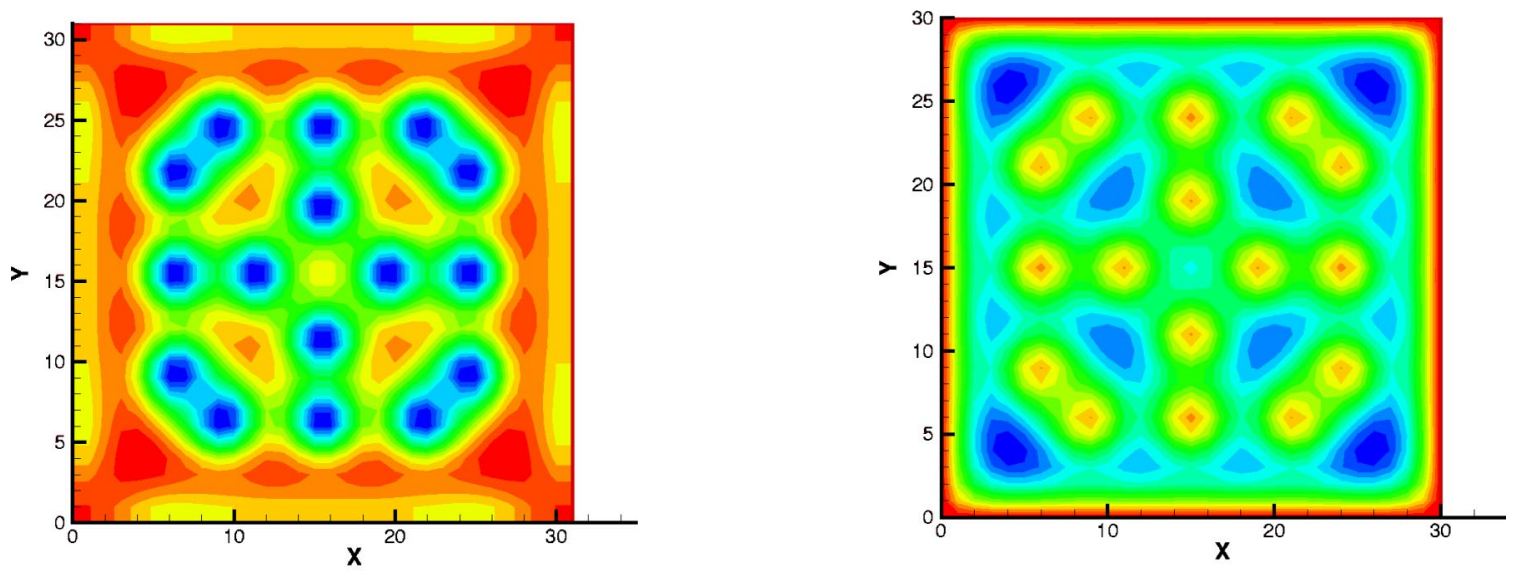

(k) $\mathrm{H}=\mathbf{2} .058$

FIG. 2. (Continued). 
TABLE I. The resultant number of vortices $n_{\infty}$ and the induced magnetic field $B$ for the applied magnetic field $H$.

\begin{tabular}{rcc}
\hline \hline$n_{\infty}$ & $H$ & $B$ \\
\hline 0 & 0.839 & 0.6163 \\
4 & 0.84 & 0.7331 \\
4 & 1.144 & 0.9779 \\
8 & 1.145 & 1.0694 \\
8 & 1.429 & 1.3022 \\
12 & 1.43 & 1.3790 \\
12 & 1.732 & 1.6288 \\
16 & 1.733 & 1.6986 \\
16 & 2.058 & 1.9719 \\
\hline \hline
\end{tabular}

configuration shows a sudden change in arrangement, even with the same number of vortices, for a slight increase of the applied field from $H=1.454$ to $H=1.455$.

\section{TIME SEQUENCE SHOWING VORTEX ENTRY DYNAMICS}

Figure 4 shows the transient development of Cooper pair density for $H=1.145$ and there are eight vortices. The perfect symmetry in the sample geometry dominates the transient process, but in the middle of the process the whole configuration makes a rotation to rearrange itself into a new configuration. (Note that time advances from 3000 to 17500 between the 8th and 9th frames.) The final result is still a square-symmetric configuration. We note that during the rotation process, the vortex configuration loses some mirror symmetries of the sample, but it still preserves the $90^{\circ}$ rotation symmetry. These transient states need not possess the full symmetry of the sample. We think this is possible because our numerical method has very weakly broken the sample symmetry. That is, the state just before the rotation is

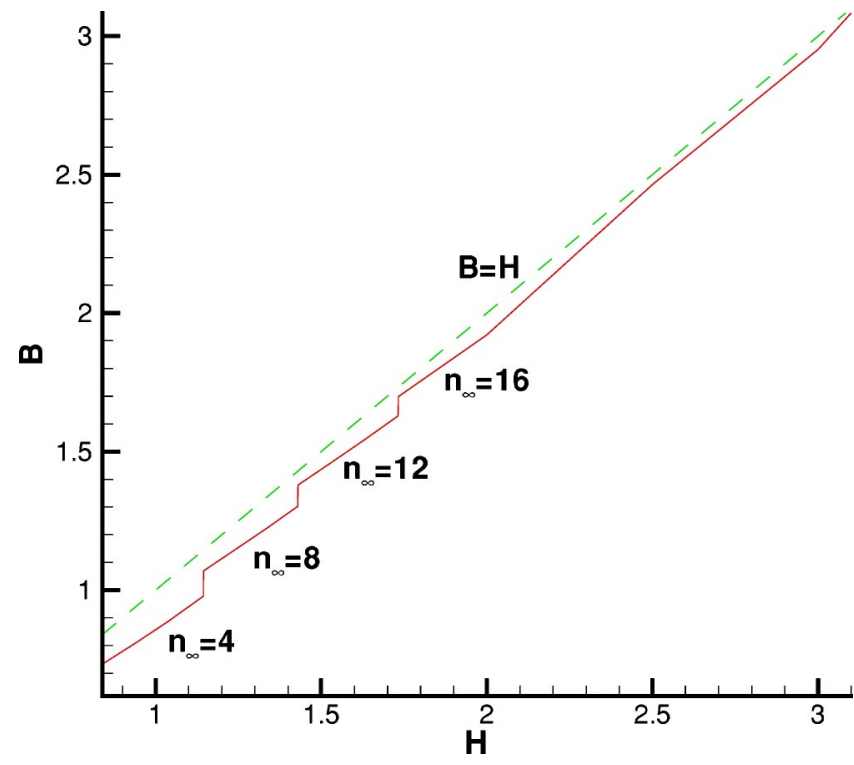

FIG. 3. The effect of applied magnetic field $H$ on the induced magnetic field $B$ averaged over the sample. metastable only within the subspace of configurations preserving the full symmetry of the sample. Thus, in a physical situation, where the sample has perfect symmetry and the temperature is sufficiently low, this rotation may take a long time to take place. For samples with imperfect symmetry this relaxation time may be shorter. Since this is a symmetryinduced qualitative property of the vortex-entry dynamics in a mesoscopic superconductor, we believe its general validity is independent of the fact that we have obtained it by solving a simplified set of TDGL equations which are not truly physical.

\section{STEADY STATES WITH REDUCED SYMMETRY AND THE EQUILIBRIUM STATE}

The previous sections present solutions for a mesoscopic type-II superconducting square cylinder with initially no vortex inside the system. The validity of such solutions requires a perfectly square sample without any defect at the boundary, and temperature sufficiently low so that thermal fluctuations are too weak to help the system find lower-energy configurations of reduced symmetry. This is an ideal condition, producing only solutions consistent with the sample symmetry. Even during the transient, the system is bound to this symmetry (except in rare cases when the transient solutions can keep only fourfold rotation symmetry but not mirror symmetries-See Fig. 4). In principle, one can reproduce this ideal system in a laboratory with special care.

In real situations, there likely exists some small defects or perturbations at the boundary, then vortices can enter the system asymmetrically to produce steady-state configurations with reduced symmetry of lower total Gibbs energy than any symmetric solution. A strong enough thermal fluctuation could also change the vortex number and rearrange the vortices to such a configuration. Previous work has taken into account these perturbations by adding a random fluctuation term to the governing equation. ${ }^{25}$ This term breaks the symmetry governing the equations by energizing the system to jump out of the local minima in energy and over the energy barrier, ${ }^{3}$ but this increases the computing time greatly. (This method is essentially "simulated annealing."17,19)

As an alternative approach, we employ perturbed initial conditions (similar to Peeters et al. ${ }^{5,7}$ ) instead of the perfectly superconducting initial condition as used in Figs. 2 and 4 . In addition, we introduce an idea to make the numerical scheme much more efficient. We have used randomly perturbed initial conditions. This can lead to final steadystate solutions with reduced symmetry and lower energies, but we find this way is very inefficient for finding the equilibrium state at any given $H$. We also tried to use a lowersymmetry configuration from such a calculation as the initial condition for a new $H$ value, but we found that the vortex number can often be trapped in an uncontrollable nonequilibrium value due to the existence of surface energy barriers against vortex entry or exit. This method of adopting an existing solution as the initial condition cannot be reliably used to find the true equilibrium state in a given system and field. (Peeters et al. changes the field in small steps to avoid this difficulty, ${ }^{26}$ but such a procedure is tedious.) 

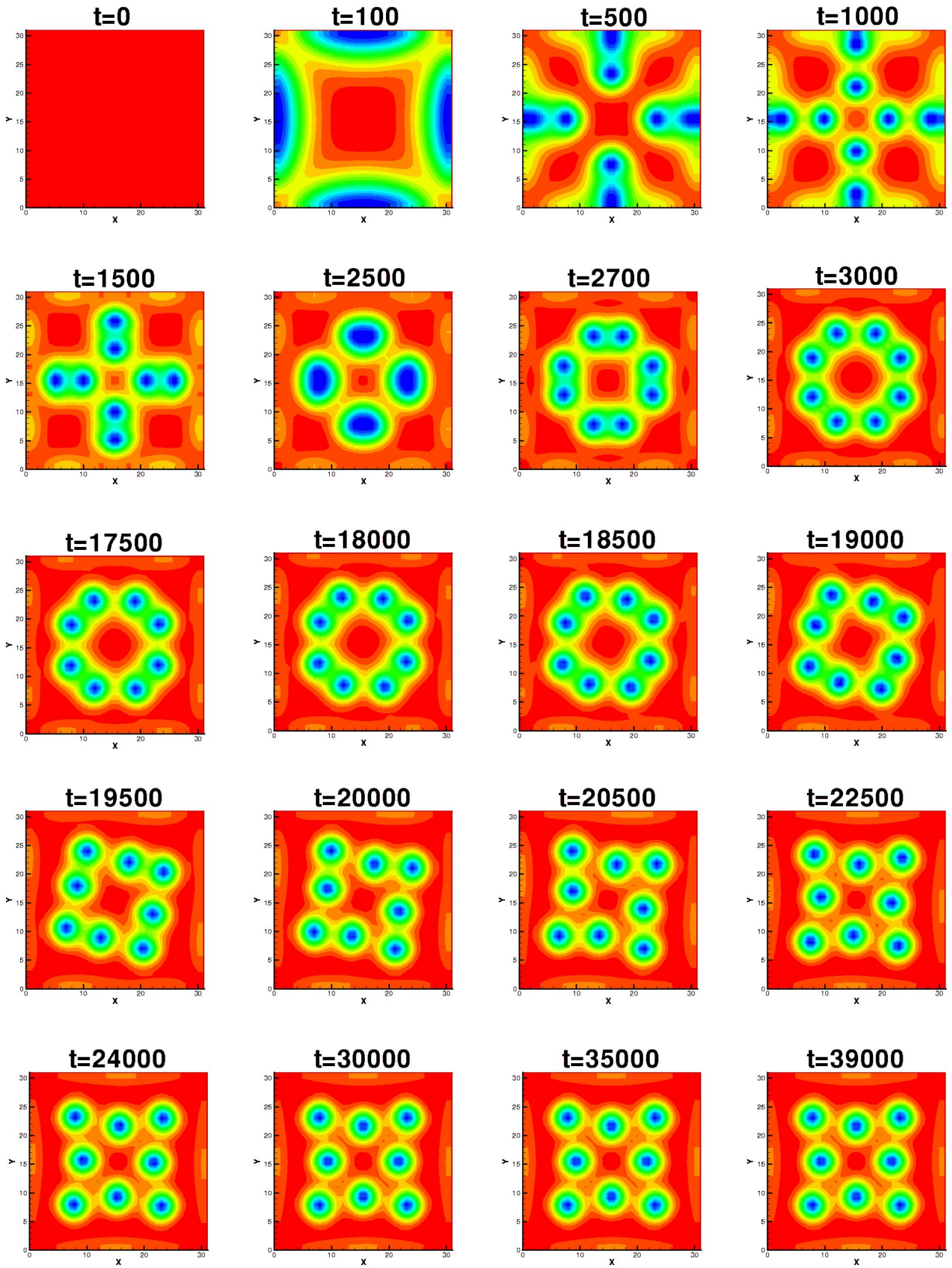

FIG. 4. Time sequence of vortex dynamics showing vortex entry for $H=1.145$. Note that the initial state (at $t=0)$ is the uniform superconducting state. 

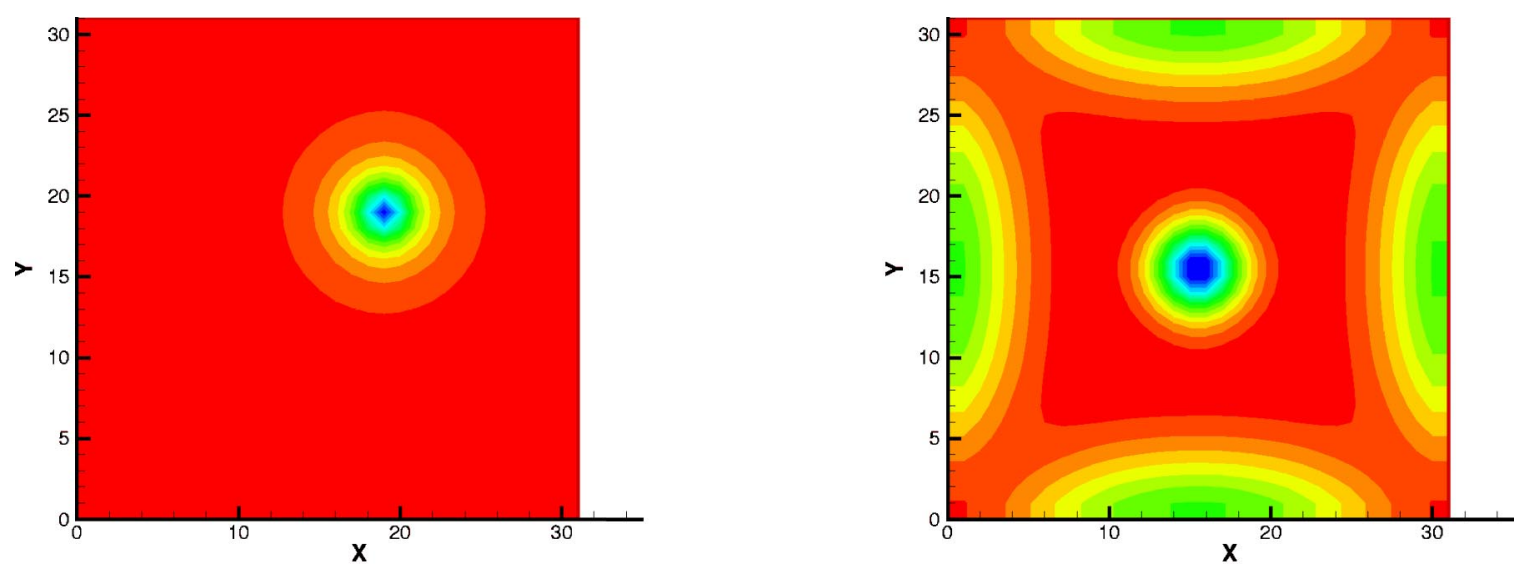

(a)
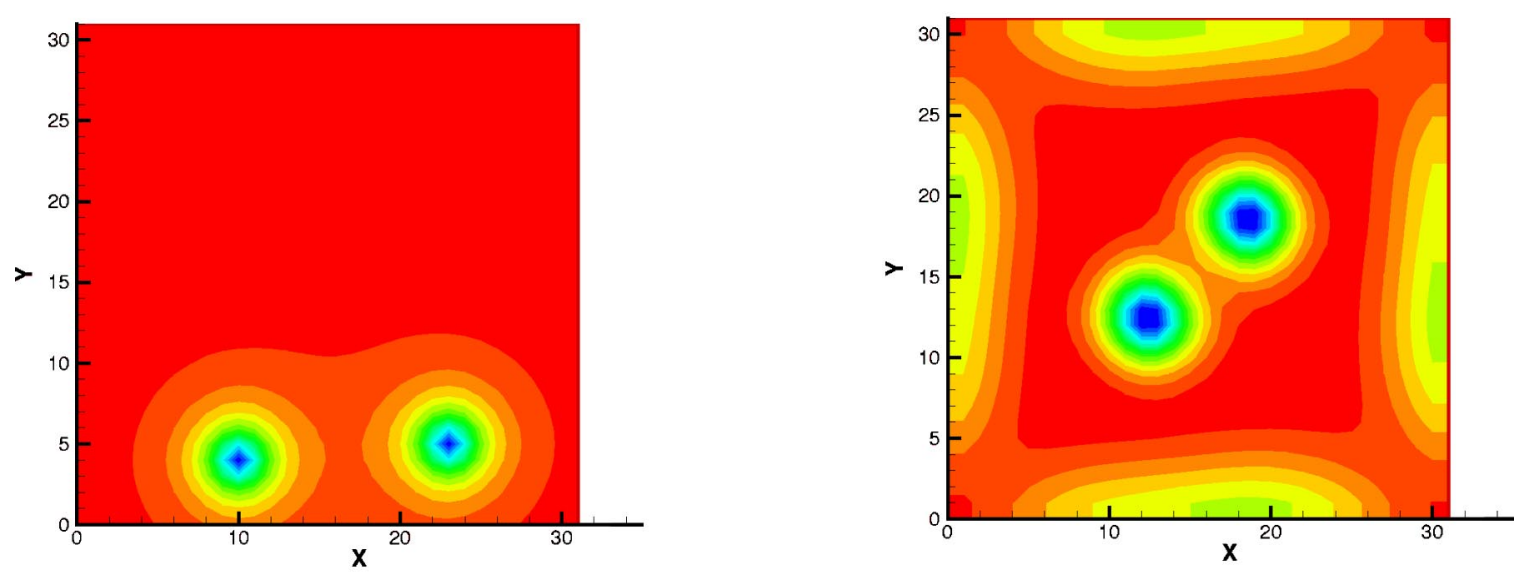

(b)
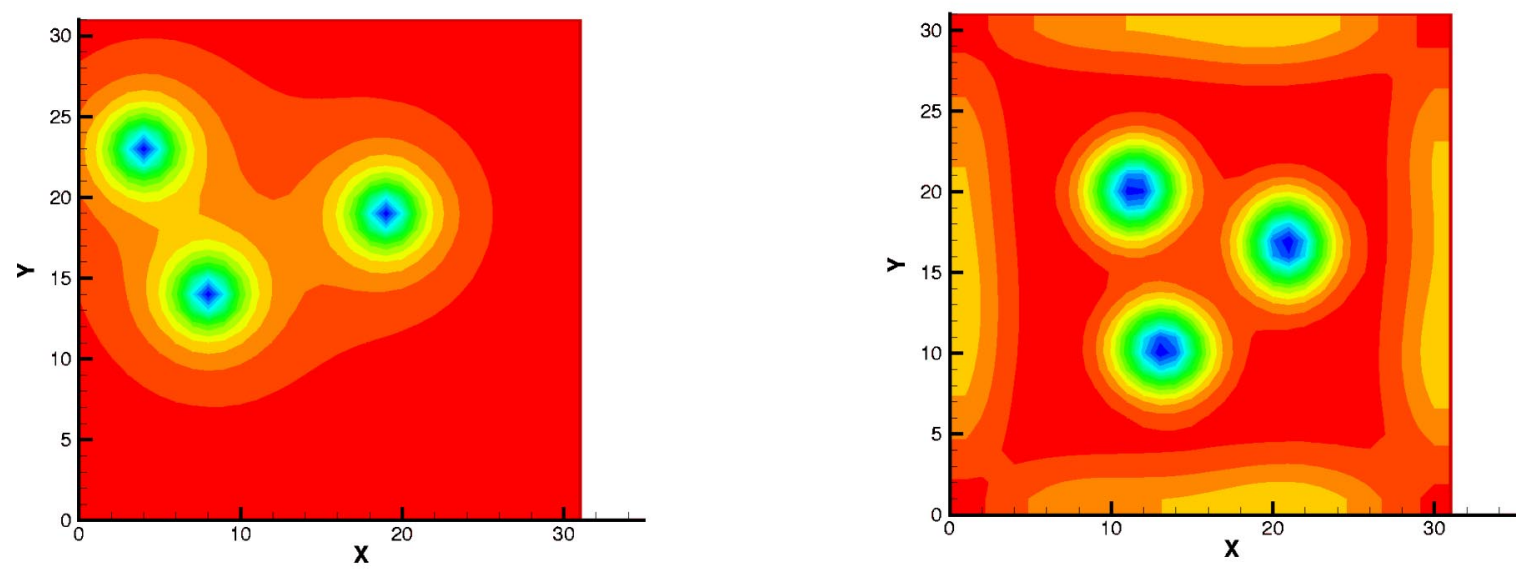

(c)

FIG. 5. The initial, random vortex configurations, and the corresponding steady-state vortex configurations they evolve to [The initial vortex configurations is shown on the left in (a)-(o), and the steady-state vortex configurations on the right in (a)-(o)]. Most of these final steady-state solutions are metastable states with a given number of trapped vortices in a field-cooled situation. The one with the lowest Gibbs energy among them may be identified as the true equilibrium state at the chosen field value, see Fig. 6. 

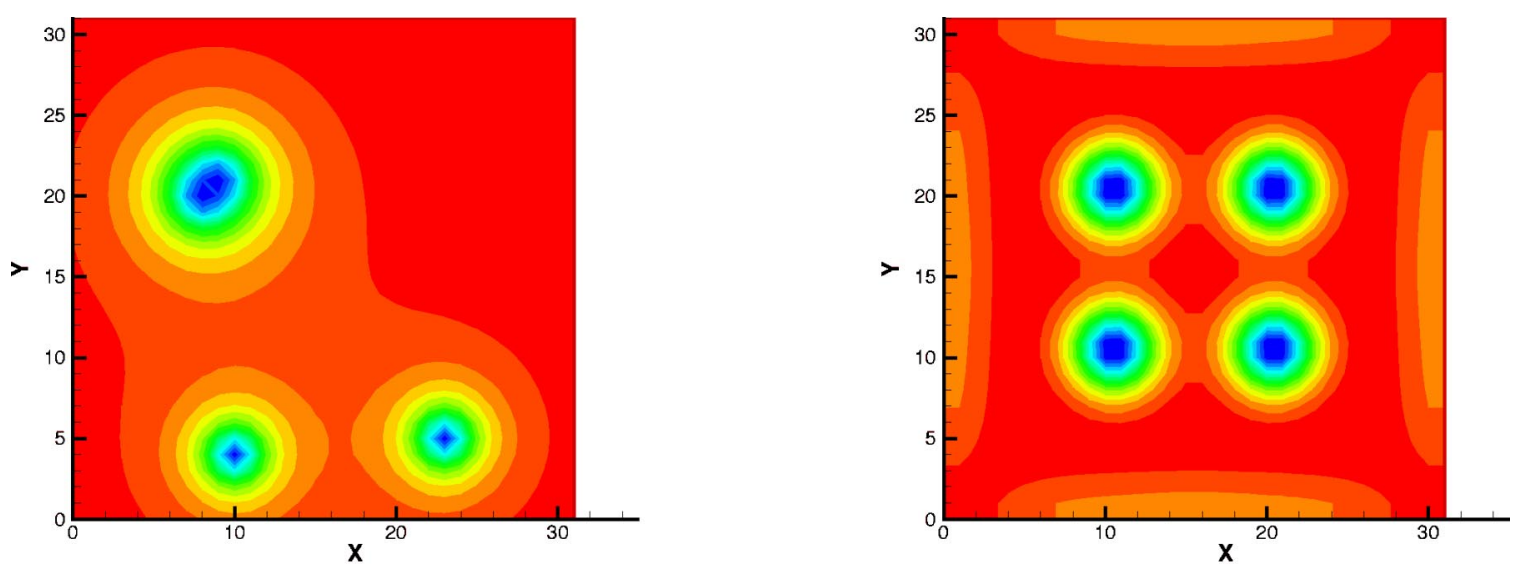

(d)
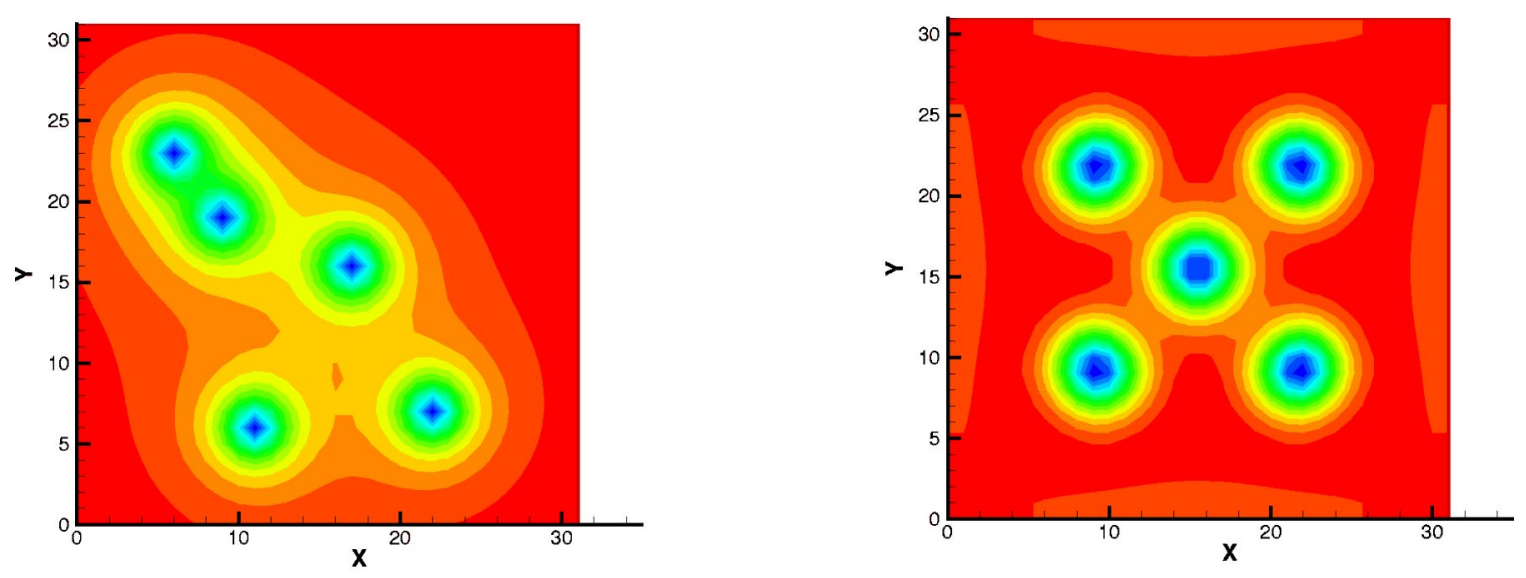

(e)
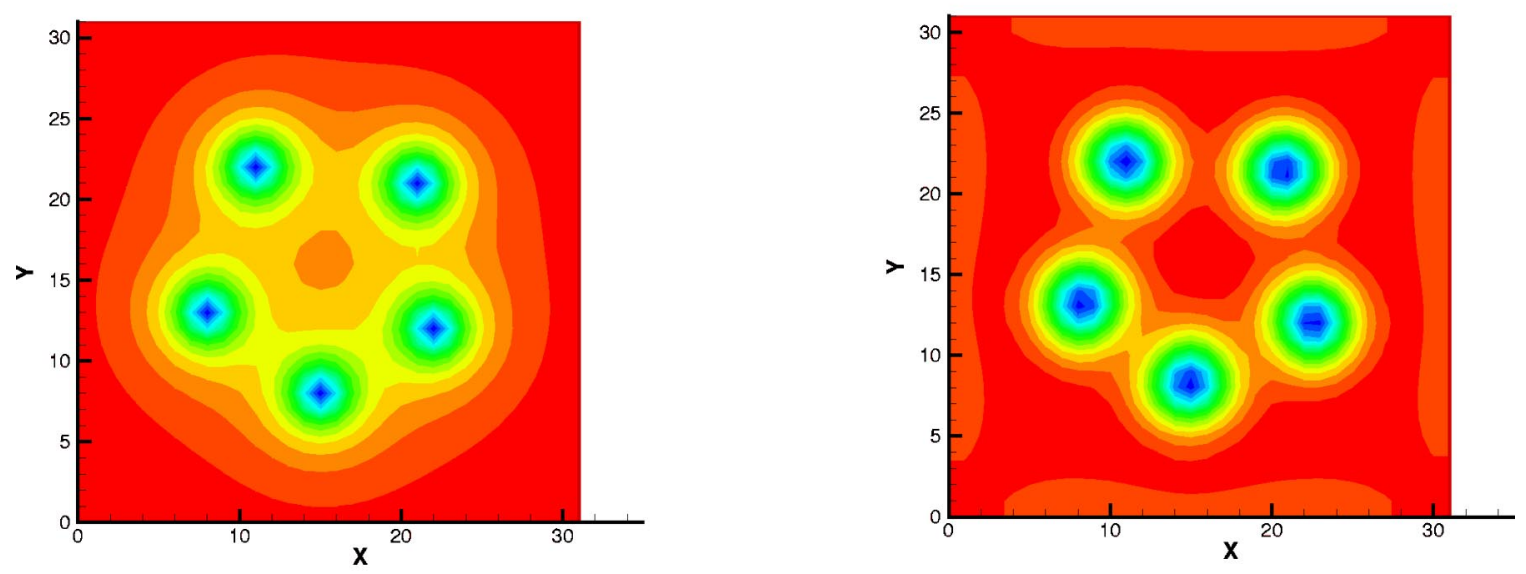

(f)

FIG. 5. (Continued). 

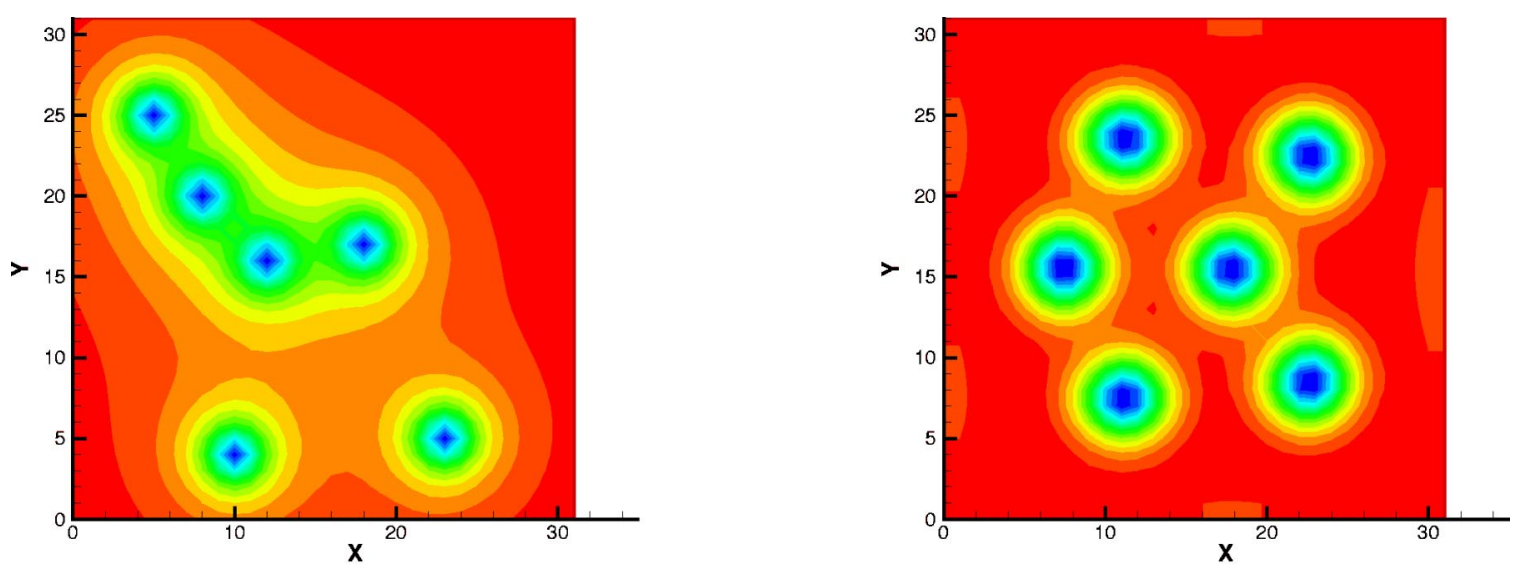

(g)
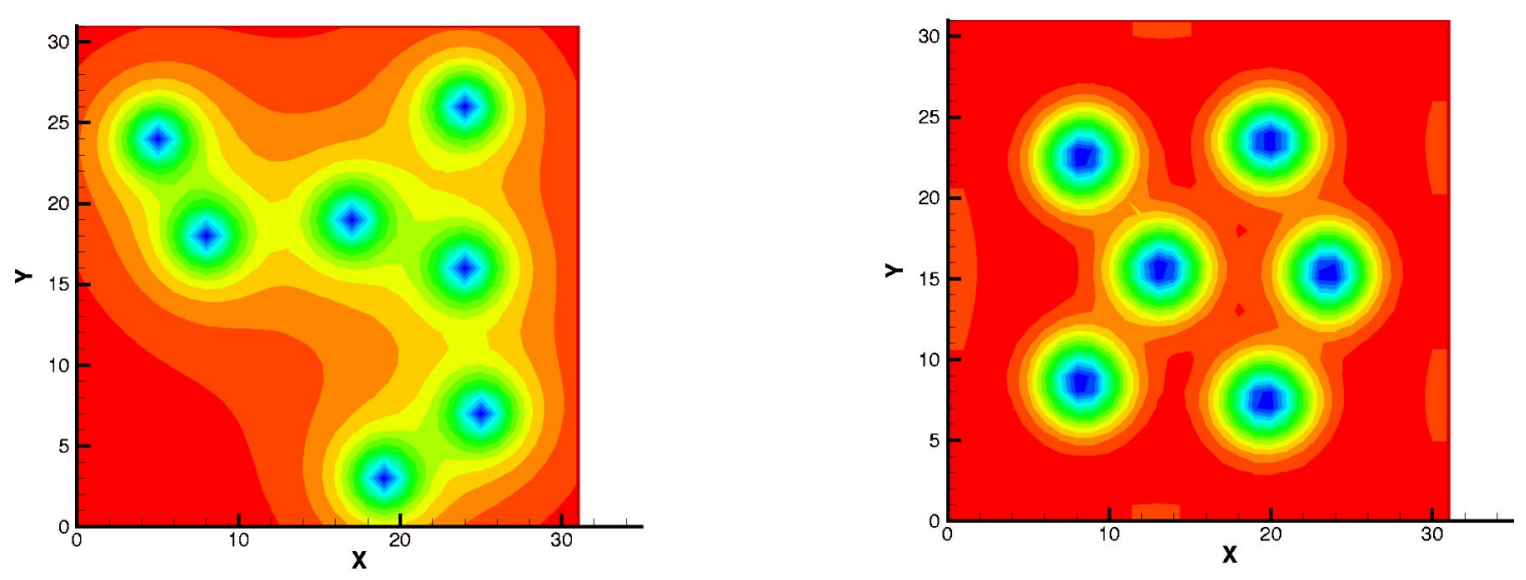

(h)
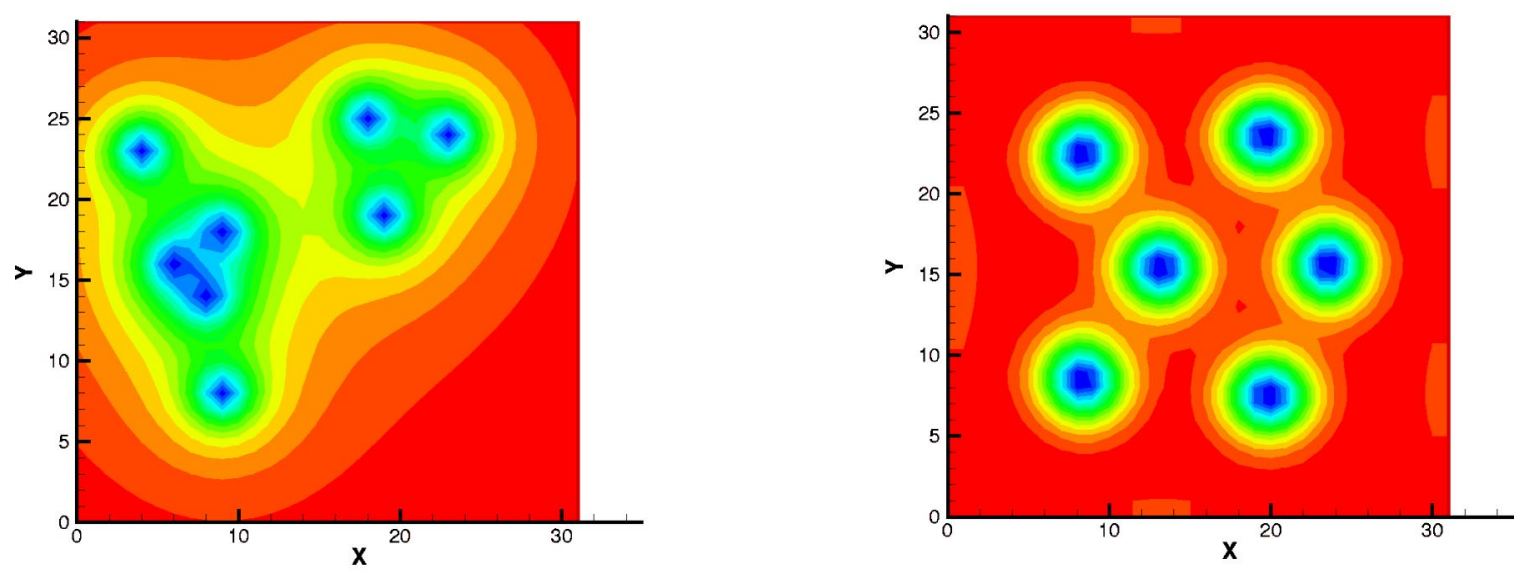

(i)

FIG. 5. (Continued). 

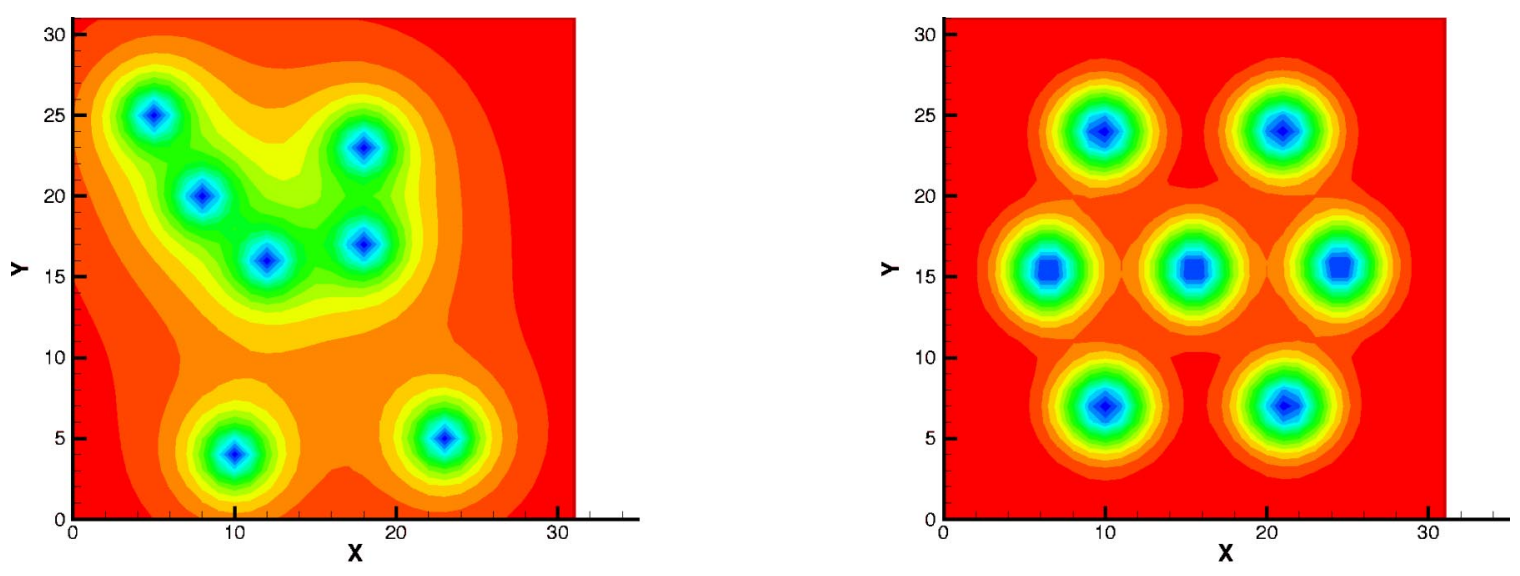

(j)
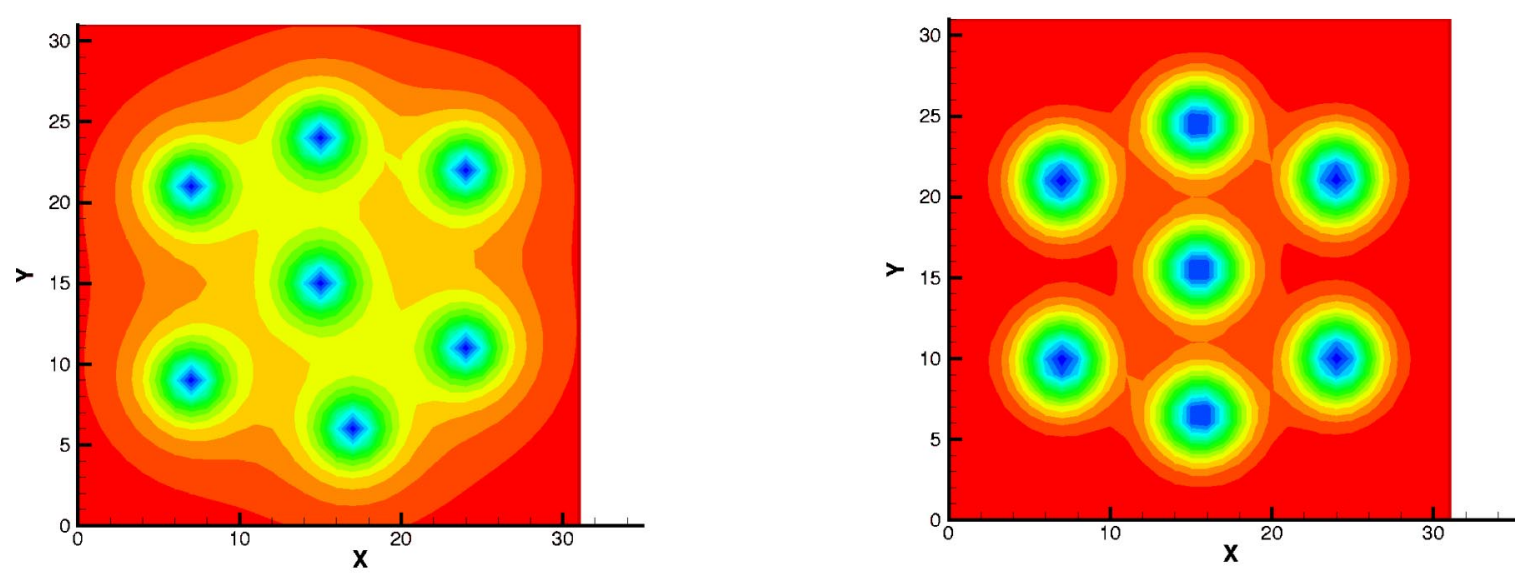

(k)
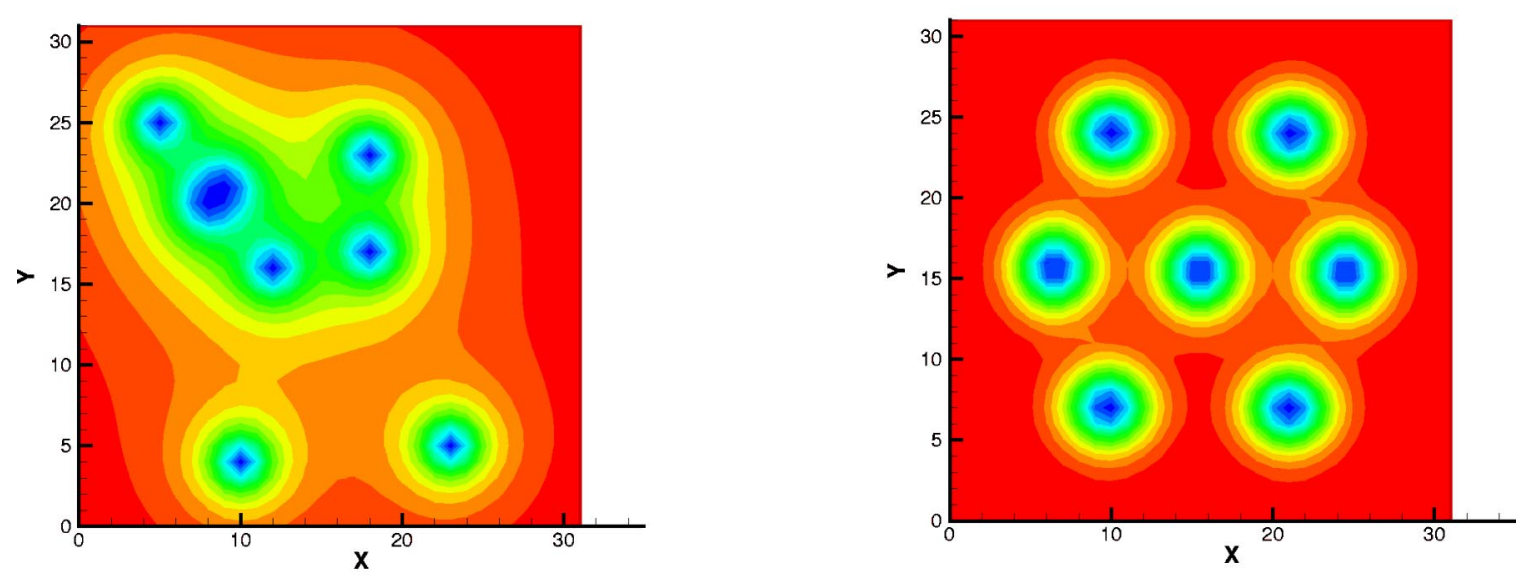

(l)

FIG. 5. (Continued). 

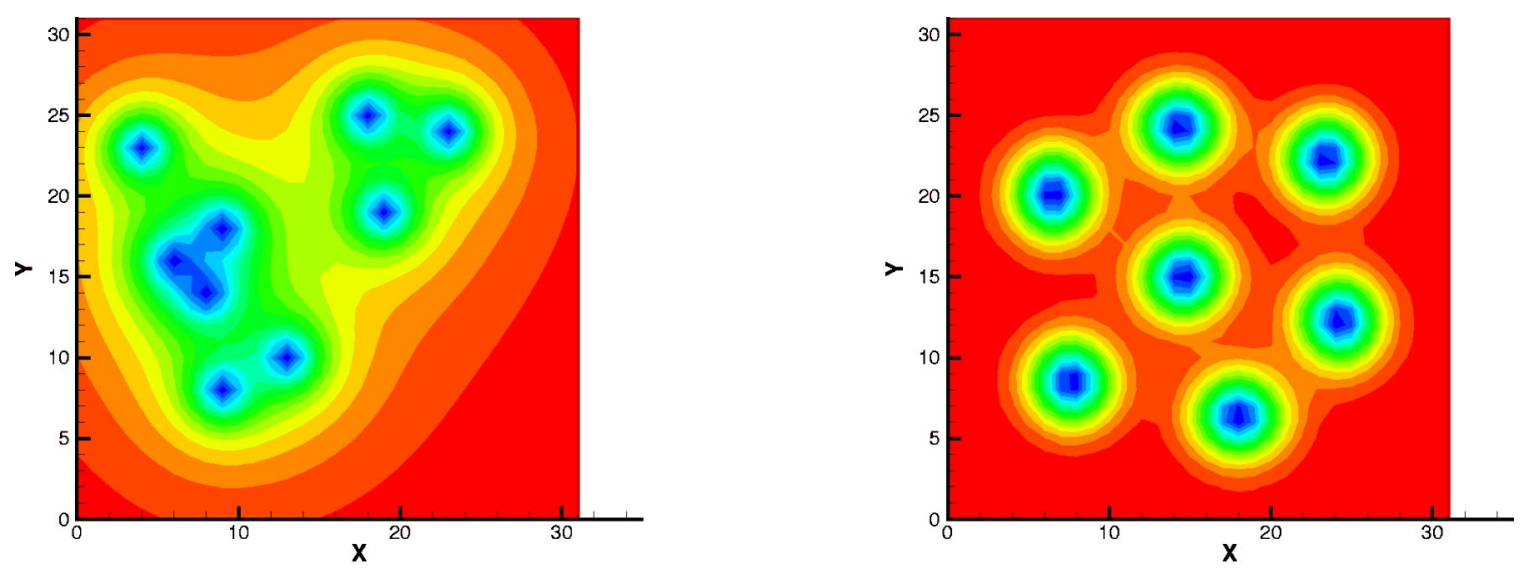

(m)
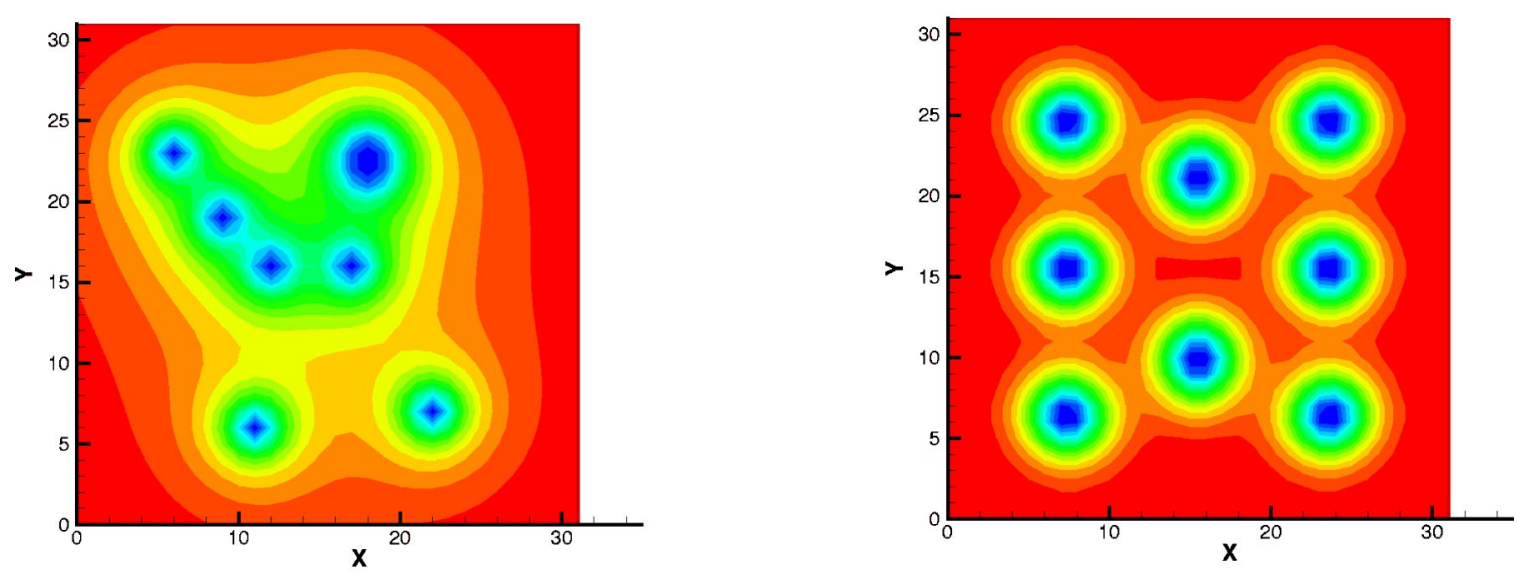

(n)
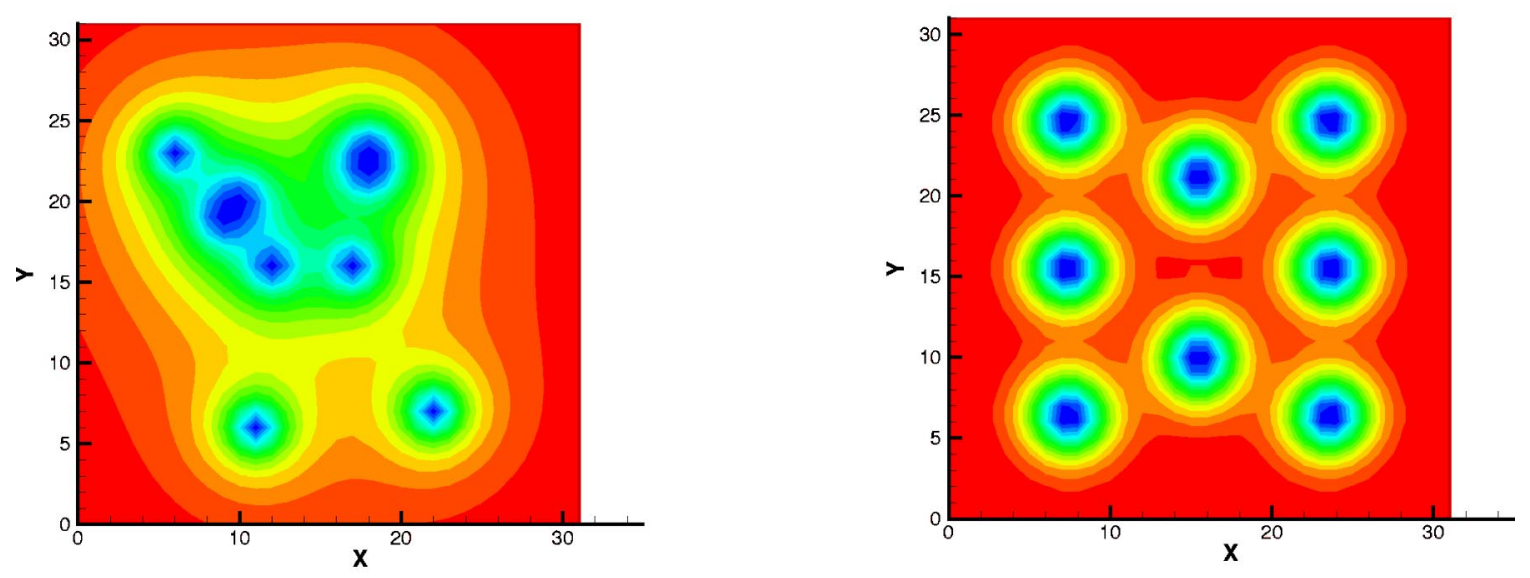

(o) 


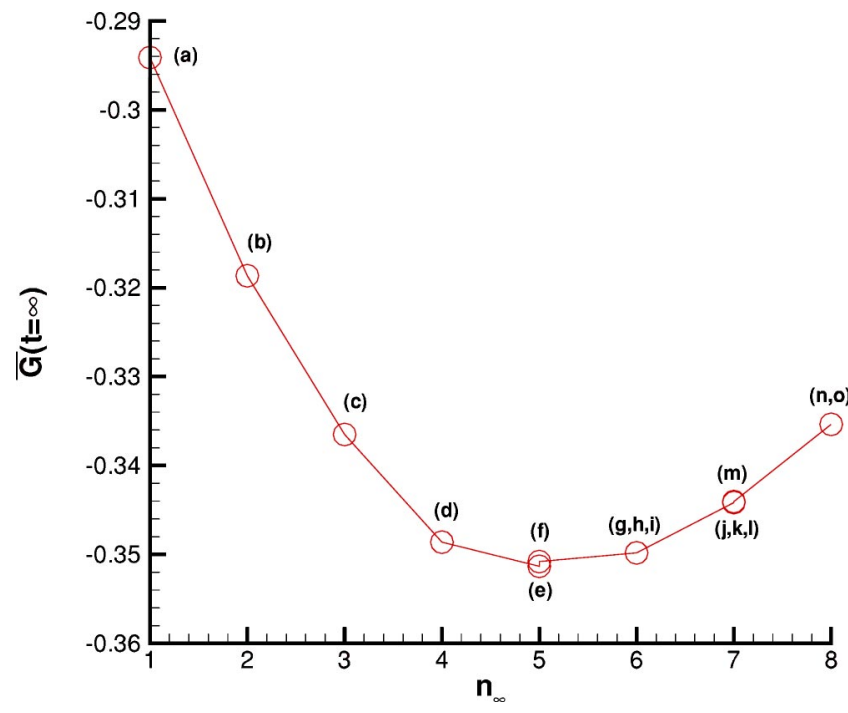

FIG. 6. The steady-state total Gibbs free energy per unit area $\bar{G}$ and the corresponding final number of vortices, $n_{\infty}$.

To obtain the true equilibrium vortex configuration at any given magnetic field without employing a simulated annealing method, we have devised a systematic approach to generate initial states with given numbers of vortices at random distributions. We start with an analytic expression as follows: for one vortex at the origin in circular coordinates $(r, \theta)$, we use the widely known approximate expression ${ }^{27-29}$

$$
\Psi(r, \theta)=\frac{r e^{i \theta}}{\sqrt{r^{2}+\kappa^{-2}}} .
$$

By converting it to Cartesian coordinates, we can move the center of the vortex to any arbitrary position $\left(x^{\prime}, y^{\prime}\right)$ by simply replacing $(x, y)$ by $\left(x-x^{\prime}, y-y^{\prime}\right)$. Denoting this expression as $\Psi_{x^{\prime}, y^{\prime}}(x, y)$, an $n$-vortex expression can be simply constructed as

$$
\Psi(x, y)=\Psi_{x_{1}, y_{1}}(x, y) \Psi_{x_{2}, y_{2}}(x, y) \cdots \Psi_{x_{n}, y_{n}}(x, y) .
$$

This expression obeys the important topological condition that the phase of $\Psi$ must increase by $2 \pi$ when any one vortex center is circumnavigated. The magnetic field inside the sample does not obey any topological condition, so it can be simply set equal to zero for the initial condition. The positions of vortices can be generated using random number generators, only if they are inside the sample. This is a straightforward idea, but it does not appear to have been employed before. We illustrate below various initial conditions used to obtain steady-state vortex configurations of any given numbers of vortices $n_{\infty}$. Comparing the total Gibbs free energy per unit area $\bar{G}$ [obtained by integrating Eq. (4) and normalized by the sample size $N_{x} h_{x} \times N_{y} h_{y}$ ] of solutions with different $n_{\infty}$, we can then determine the equilibrium vortex number and configuration. For illustrative purposes, we consider the case $H=0.840$. Figure 5 shows the initial conditions for $\Psi$ with 1-8 randomly placed (artificial) vortices [the left figures in (a)-(o)], and the steady-state vortex
TABLE II. The steady-state total Gibbs free energy $\bar{G}$ and \% error in $\bar{G}$ for changing grid size for artificial vortex initial condition $(H=0.84)$ and uniformly superconducting initial condition $(H=1.733)$.

\begin{tabular}{lcccc}
\hline \hline \multirow{2}{*}{ Grid } & \multicolumn{2}{c}{ Artificial IC } & \multicolumn{2}{c}{ Uniform IC } \\
& $\bar{G}$ & \% error & $\bar{G}$ & \% error \\
\hline $16 \times 16$ & -0.3487 & 0.71 & -0.1814 & \multirow{2}{*}{14.1} \\
$32 \times 32$ & -0.3513 & & -0.2069 & \\
$32 \times 32$ & -0.3513 & 0.17 & -0.2069 & \multirow{2}{*}{0.41} \\
$64 \times 64$ & -0.3519 & & -0.2078 & \\
\hline \hline
\end{tabular}

configurations they evolve to [the right figures in (a)-(o)]. Indeed in Figs. 5(i), 5(1), 5(m), 5(o), the initial number of vortices is not always the final number. This is because the energy of the initial state is high when vortices are initially very close to each other, in which case there is enough energy to overcome the surface barrier to eject excess vortices. In particular, comparison of the initial and final states in Fig. 5(h) reveals that one vortex was expelled during the evolution. This is because the surface barrier was weakened, perhaps by one vortex in the initial state being close to the edge of the sample, and the total number of vortices being too high compared with the final equilibrium vortex number. The corresponding total Gibbs free energies $\bar{G}$ of these steady states are plotted in Fig. 6 as a function of the final vortex number $n_{\infty}$. The minimum-energy configuration at $n_{\infty}=5$ is seen to display the square symmetry of a five-vortex configuration with a vortex in the center. Grid independence checks were performed, and Fig. 7(a) shows this five-vortex case using grids of $64 \times 64$, and in Fig. 7(b) a uniformly superconducting initial condition with $H=1.733$ corresponding to Fig. 2(j). Comparison of Fig. 5(e) (32×32 grid) with Fig. 7 (a) $(64 \times 64$ grid) reveals close agreement. Similarly comparison of Fig. 2(j) and Fig. 7(b) shows close agreement. A more stringent grid independence test was to consider the resultant total Gibbs free energy for each grid, and given in Table II. Inspection of the table reveals convergence to less than a $1 \%$ change when moving to different grids. The percent error was defined as: $\%$ error $=\left|\bar{G}_{64 \times 64}-\bar{G}_{32 \times 32}\right|$ $\times 100 /\left|\bar{G}_{32 \times 32}\right|$, and similarly for other cases. These grid independence results support our use of $32 \times 32$ grids for this study. The large error of $14.1 \%$ for the uniform initial condition case in Table II was associated with a decrease from 16 vortices to 12 when the grid was coarsened from 32 $\times 32$ to $16 \times 16$. The reduction in resolution caused the vortex number to cross the $H$ threshold and produced a 12 vortex configuration. Our exploration of finer grids ensured that we determined an accurate $H$ threshold, and not one dependent on the grid.

Although we have not yet applied this scheme to other field values of $H$, the method we have devised to find the equilibrium vortex configurations for a given size and shape of the sample, and different values of the external magnetic field should now be clear. 


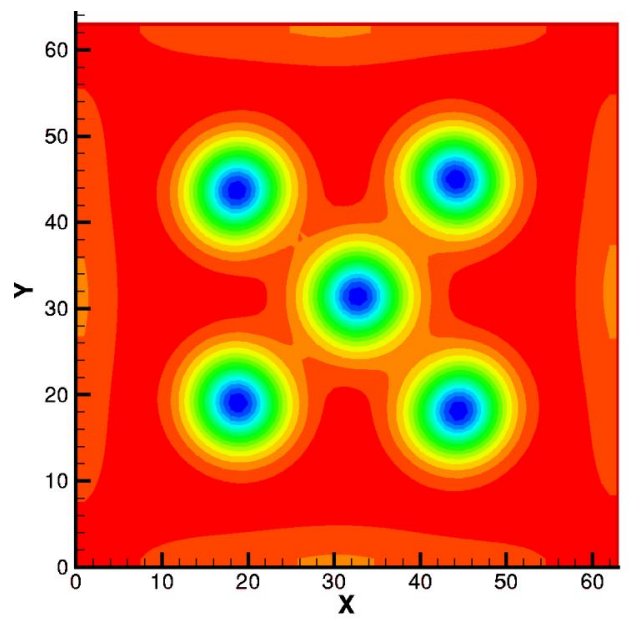

(a)

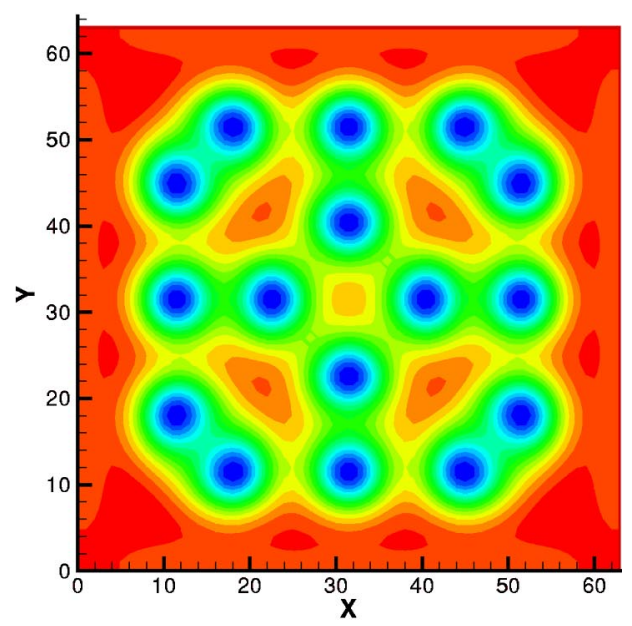

(b)

FIG. 7. Examples of the steady-state vortex configurations for larger grid size $(64 \times 64)$. (a) Artificial vortex initial condition under $H$ $=0.84$, (b) uniformly superconducting initial condition under $H=1.733$.

\section{SUMMARY AND CONCLUSION}

A numerical scheme to study the mixed states in a mesoscopic type-II superconducting square cylinder in a longitudinal external magnetic field $H$ has been developed. It is based on solving a set of simplified time-dependent Ginzburg-Landau equations. We have first applied this scheme to the case of field penetration into a zero-fieldcooled sample. Case studies for various values of the external magnetic field were presented. Contour plots of the Cooper pair density, and the induced magnetic field inside the sample, display the magnetic vortex solution first discovered by Abrikosov, but in a small sample the vortex arrangement is not simply triangular. Giant vortices and antivortices are not found in this study, unlike previous studies of type-I mesoscopic thin films. (But at sufficiently high magnetic field we still expect the system to favor a single giant vortex at the center as it goes into a surface superconducting state, but only if the sample is not too small.) Since we start the solution with a uniformly superconducting initial condition, and the sample has perfect square symmetry, both the number of vortices and their steady-state configurations are governed by the square sample geometry. Changes in the configuration and the number of vortices occur as $H$ is varied through first-order configurational phase transitions, similar to those found earlier, but different in detail. This phase transition characteristic is confirmed by the contour plots, and jumps in the values of the induced magnetic field $B$ at certain discrete $H$ values. A time sequence shows that the system passes through intermediate configurations, and remains in some of them for a long time. Eventually the system settles down to the steady-state configuration, which corresponds to the lowest-Gibbs-energy configuration consistent with the symmetry constraints to the vortex number and configuration. True equilibrium states would appear in actual samples when there are symmetry-breaking surface defects forming vortex-nucleation centers, or when thermal fluctuations are sufficiently strong to move the system out of metastable states, but not too strong to melt the vortex lattice. We could have determined these true equilibrium states by adding additional terms in the equations to simulate thermal fluctuations, but here we have devised a different approach which we believe is more efficient. We introduce a way to generate analytic initial states of prescribed numbers of vortices, but allow their positions to be random. They evolve to steadystate vortex arrangements of all possible vortex numbers near the equilibrium number, from which we can compare total Gibbs energy to determine the equilibrium vortex number and configuration. In this way, we avoid the problem of surface and bulk energy barriers, which can trap the system in nonequilibrium vortex numbers and configurations-an undesirable situation which usually happens if one chooses the initial state randomly without controlling the vorticity quantum number $L$.

\section{ACKNOWLEDGMENTS}

$\mathrm{Hu}$ wishes to acknowledge the support from the Texas Center for Superconductivity and Advanced Materials at the University of Houston. Andrews acknowledges support from the Texas A\&M University through the Telecommunications and Informatics Task Force. 
${ }^{1}$ P. G. de Gennes, Superconductivity in Metals and Alloys (W. A. Benjamin, New York, 1966, reprinted by Addison-Wesley, Reading, MA, 1989).

${ }^{2}$ S.J. Chapman, SIAM Rev. 42, 555 (2000).

${ }^{3}$ M. Tinkham, Introduction to Superconductivity (McGraw-Hill, New York, 1996).

${ }^{4}$ A.A. Abrikosov, Zh. Éksp. Teor. Fiz. 32, 1442 (1957) [Sov. Phys. JETP 5, 1174 (1957)].

${ }^{5}$ B.J. Baelus and F.M. Peeters, Phys. Rev. B 65, 104515 (2002).

${ }^{6}$ V.A. Schweigert and F.M. Peeters, Phys. Rev. B 57, 13817 (1998).

${ }^{7}$ V.A. Schweigert, F.M. Peeters, and P. Singha Deo, Phys. Rev. Lett. 81, 2783 (1998).

${ }^{8}$ P. Singha Deo, V.A. Schweigert, F.M. Peeters, and A.K. Geim, Phys. Rev. Lett. 79, 4653 (1997).

${ }^{9}$ J. Bonca and V.V. Kabanov, Phys. Rev. B 65, 012509 (2001).

${ }^{10}$ V.R. Misko, V.M. Fomin, J.T. Devereese, and V.V. Moshchalkov, Physica C 369, 361 (2002).

${ }^{11}$ K. Geim, S.V. Dubonos, J.J. Palacios, I.V. Grigorieva, M. Henini, and J.J. Schermer, Phys. Rev. Lett. 85, 1528 (2000).

${ }^{12}$ V.R. Misko, V.M. Fomin, J.T. Devreese, and V.V. Moshchalkov, Phys. Rev. Lett. 90, 147003 (2003).

${ }^{13}$ In this brief survey of literature, we have not included solutions of linearized GL equations to study the vortex configurations near the phase boundary, studies of vortex configurations in the presence of pinning center(s), and studies based on the London approximation.

${ }^{14}$ G.W. Crabtree, D.G. Gunter, H.G. Kaper, A.E. Koshelev, G.K. Leaf, and V.M. Vinokur, Phys. Rev. B 61, 1446 (2000).

${ }^{15}$ K.J.M. Moriarty, E. Myers, and C. Rebbi, Comput. Phys. Commun. 54, 273 (1989).

${ }^{16}$ H.G. Kaper and M.K. Kwong, J. Comput. Phys. 119, 120 (1995).

${ }^{17}$ W. H. Press, B. P. Flannery, S. A. Teukolsky, and W. T. Vetterling,
Numerical Recipes (Cambridge University Press, Cambridge, 1989), Sec. 10.9.

${ }^{18}$ M.M. Doria, J.E. Gubernatis, and D. Rainer, Phys. Rev. B 41, 6335 (1990).

${ }^{19}$ For a larger sample in a sufficiently large magnetic field, we can occasionally obtain configurations with a reduced symmetry when solving the equations starting with the uniform superconducting state, but this is very rare, and very difficult to control. We think that this is because the numerical program does not observe the perfect square symmetry, but this violation of symmetry is a weak one. This way to get an asymmetric steady state is not reliable, so we do not recommend its use to find any asymmetric equilibrium state.

${ }^{20}$ As far as we know, previous approaches used random initial conditions with no control of $L$, so the magnetic field must be changed in very small steps to obtain speedy convergence. If so, the present scheme represents an important improvement, which allows direct implementation at any applied field. Note that the scheme also applies to the situation when a given distribution of pinning centers exist in the system or on its surface.

${ }^{21}$ Q. Du, M.D. Gunzburger, and J.S. Peterson, SIAM Rev. 34, 54 (1992).

${ }^{22}$ E. Coskun and M.K. Kwong, Nonlinearity 10, 579 (1997).

${ }^{23}$ S.L. Adler and T. Piran, Rev. Mod. Phys. 56, 1 (1984).

${ }^{24}$ For a different way to discretize the problem without involving staggered grid, see Z.D. Wang and C.R. Hu, Phys. Rev. B 44, 11 918 (1991).

${ }^{25}$ R. Kato, Y. Enomoto, and S. Maekawa, Phys. Rev. B 47, 8016 (1993).

${ }^{26}$ F. M. Peeters (private communication).

${ }^{27}$ A. Schmid, Phys. Kondens. Mater. 5, 302 (1966).

${ }^{28}$ C.-R. Hu and R.S. Thompson, Phys. Rev. B 6, 110 (1972).

${ }^{29}$ J.R. Clem, J. Low Temp. Phys. 18, 427 (1975). 